\title{
Resonance Treatment using Pin-Based Pointwise Energy Slowing-Down Method
}

\author{
Sooyoung Choi ${ }^{\mathrm{a}}$, Changho Lee ${ }^{\mathrm{b}}$, Deokjung Lee ${ }^{\mathrm{a}, *}$ \\ ${ }^{a}$ Ulsan National Institute of Science and Technology, 50 UNIST-gil, Ulsan 44919, Korea \\ ${ }^{\mathrm{b}}$ Argonne National Laboratory, 9700 S. Cass Avenue, Argonne, IL 60439, United States \\ ${ }^{*}$ Corresponding author. Tel.: +82-52-217-2940, +82-10-2863-6255, fax.: +82-52-217-3008. \\ E-mail addresses: csy0321@ unist.ac.kr (S. Choi), clee@anl.gov (C. Lee), \\ deokjung@unist.ac.kr (D. Lee)
}

\begin{abstract}
A new resonance self-shielding method using a pointwise energy solution has been developed to overcome the drawbacks of the equivalence theory. The equivalence theory uses a crude resonance scattering source approximation, and assumes a spatially constant scattering source distribution inside a fuel pellet. These two assumptions cause a significant error, in that they overestimate the multi-group effective cross sections, especially for ${ }^{238} \mathrm{U}$. The new resonance self-shielding method solves pointwise energy slowing-down equations with a sub-divided fuel rod. The method adopts a shadowing effect correction factor and fictitious moderator material to model a realistic pointwise energy solution. The slowingdown solution is used to generate the multi-group cross section. With various light water reactor problems, it was demonstrated that the new resonance self-shielding method significantly improved accuracy in the reactor parameter calculation with no compromise in computation time, compared to the equivalence theory.
\end{abstract}

Keywords: Reactor physics, resonance treatment, resonance self-shielding, equivalence theory, light water reactor, slowing-down. 


\section{Introduction}

Equivalence theory has been widely used for resonance treatment methods [1,2]. The equivalence theory gives a reasonable solution within a short computation time. Because of this many lattice physics codes adopt the equivalence theory to generate the effective crosssection (XS) [Error! Reference source not found.,4]. There have been a lot of research into equivalence theory to improve the accuracy of multi-group effective XS and the applicability for general geometry [Error! Reference source not found.,5,6].

Recently, Cao reported that the multi-group ${ }^{238} \mathrm{U}$ absorption XS from the equivalence theory tends to be overestimated [7]. He concluded that the flux from the narrow resonance (NR) approximation in the equivalence theory is problematic, and suggested an improved resonance treatment method using a pre-generated look-up table of integrated flux like the multi-group effective XS. Yamamoto focused on the reaction rate preservation between the ultrafine group calculation and the multi-group effective XS calculation with multi-term rational approximation, and suggested an improved derivation [8]. The two researches were performed to correct an overestimation of ${ }^{238} \mathrm{U}$ absorption XS.

The overestimation of the ${ }^{238} \mathrm{U}$ effective XS is addressed in this paper. This work figures out that resonance scattering causes the overestimation. In the equivalence theory, the resonance scattering source is approximated by the intermediate resonance (IR) approximation, and the resonance scattering XS is usually neglected in the resonance treatment process. However, for the nuclides (i.e., ${ }^{238} \mathrm{U}$ ) which have very large resonance scattering XSs, the equivalence theory causes a significant error in the effective XS because of the following reasons. First, the expression for the effective XS is derived from the multiterm rational approximation along with the NR or IR approximation. The derivation is formulated based on the IR approximation, but the final effective XS is calculated as a linear combination of the effective XSs generated from multiple dilution systems. This discrepancy between the derivation and usage of the XS look-up table can cause an error in the effective XS. Second, the equivalence theory cannot treat the spatial distribution of the effective XS inside the fuel pellet. Since the theory is based on the averaged effective XS of the fuel lump, the spatial self-shielding and spatial distribution of the scattering source cannot be considered properly. There are several methods which can consider spatial self-shielding inside the fuel pellet $[9,10,11]$. However, as the methods are based on the pellet-averaged effective XS [10] 
or the pellet-averaged escape probability approximation $[9,11]$ with the IR approximation, the resonance scattering source cannot be accounted for accurately.

This paper suggests a new resonance self-shielding method using the pointwise energy slowing-down solution in order to resolve the two problems described above. The new method computes the collision probabilities inside the fuel pellet as a function of the total XS, and then solves pointwise energy slowing-down equations based on the pin-cell. The shadowing effect correction factor and fictitious moderator are introduced to derive a realistic pointwise energy slowing down equation. The multi-group effective XS is calculated using the pointwise flux spectrum. The new method is verified with various light water reactor (LWR) problems and shows significant improvements in the accuracy of the effective XS and the multiplication factor.

\section{Overestimation of ${ }^{238} \mathrm{U}$ Cross Sections}

The conventional equivalence theory is summarized in Section 2.1, and numerical test results are described in Section 2.2 in order to show the overestimation of ${ }^{238} \mathrm{U} X S$ with the equivalence theory.

\subsection{Equivalence Theory}

A transport equation with collision probabilities for the two-region problem is

$$
\Sigma_{t, F}(E) \phi_{F}(E) V_{F}=P_{F F}(E) V_{F} Q_{s, F}(E)+P_{M F}(E) V_{M} Q_{s, M}(E)
$$

where

$$
\left\{\begin{array}{l}
Q_{s, F}(E)=\sum_{r \in F} N^{r} \int_{E}^{E / \alpha^{r}} \sigma_{s}^{r}\left(E^{\prime}\right) \phi_{F}\left(E^{\prime}\right) \frac{1}{1-\alpha^{r}} \frac{d E^{\prime}}{E^{\prime}} \\
Q_{s, M}(E)=\sum_{r \in M} N^{r} \int_{E}^{E / \alpha^{r}} \sigma_{s}^{r}\left(E^{\prime}\right) \phi_{M}\left(E^{\prime}\right) \frac{1}{1-\alpha^{r}} \frac{d E^{\prime}}{E^{\prime}}
\end{array},\right.
$$

and $F$ is the index of fuel; $M$ is the index of moderator; $\Sigma_{t, F}(E)$ is the total XS of fuel; $\phi_{F}(E)$ is the flux in fuel; $V_{F}$ is the volume of fuel, $P_{F F}(E)$ is the first flight fuel collision 
probability (or fuel-to-fuel collision probability); $P_{M F}(E)$ is the collision probability from $M$ to $F ; N^{r}$ is the number density of nuclide $r ; \sigma_{s}^{r}(E)$ is the scattering XS of nuclide $r ; \alpha^{r}=\left(1-A^{r}\right)^{2} /\left(1+A^{r}\right)^{2}$; and $A^{r}$ is the mass of nuclide $r$.

The scattering source from the nuclide in the fuel material is written with the IR approximation as follows:

$$
\int_{E}^{E / \alpha^{r}} \sigma_{s}^{r}\left(E^{\prime}\right) \phi_{F}\left(E^{\prime}\right) \frac{1}{1-\alpha^{r}} \frac{d E^{\prime}}{E^{\prime}} \approx \lambda^{r} \sigma_{p}^{r} \frac{1}{E}+\left(1-\lambda^{r}\right) \sigma_{s}^{r}(E) \phi_{F}(E)
$$

where $\lambda^{r}$ is the IR parameter; and $\sigma_{p}^{r}$ is potential XS of the nuclide $r$.

The scattering source from the nuclide in the non-fuel material (or moderator) is approximated as follows:

$$
\int_{E}^{E / \alpha^{r}} \sigma_{s}^{r}\left(E^{\prime}\right) \phi_{M}\left(E^{\prime}\right) \frac{1}{1-\alpha^{r}} \frac{d E^{\prime}}{E^{\prime}} \approx \lambda^{r} \sigma_{p}^{r} \frac{1}{E}
$$

Although the IR parameter is introduced to approximate the resonance scattering source, the IR parameter considers the average energy loss in the collision as well as the width of resonance peak in the practical lattice physics calculation [1]. This is why the IR parameter is used in approximating the scattering source of the moderator, even though the moderator does not usually have a resonance. Eq. (1) is rewritten by using the approximate scattering source and a reciprocity theorem in Eq. (6) as follows:

$$
\Sigma_{t, F}(E) \phi_{F}(E)=P_{F F}(E)\left[\lambda_{F} \Sigma_{p, F} \frac{1}{E}+\left(1-\lambda_{F}\right) \Sigma_{s, F}(E) \phi_{F}(E)\right]+P_{F M}(E) \Sigma_{t, F}(E) V_{F} \frac{1}{E},
$$

where $\lambda_{X} \Sigma_{p, X}=\sum_{r \in X} \lambda^{r} N^{r} \sigma_{p}^{r},(X=F$ or $M)$; and the reciprocity theorem is

$$
P_{F M}(E) \Sigma_{t, F}(E) V_{F}=P_{M F}(E) \Sigma_{t, M} V_{M} \approx P_{M F}(E) \lambda_{M} \Sigma_{p, M} V_{M}
$$

Then, the fuel-to-fuel collision probability is approximated by rational equation as

$$
P_{F F}(E)=1-P_{F M}(E)=\sum_{n=1}^{N} \frac{\beta_{n} \Sigma_{t, F}(E)}{\Sigma_{t, F}(E)+\alpha_{n} \Sigma_{e}},
$$


where $N$ is the number of rational expressions; $\alpha_{n}$ and $\beta_{n}$ are coefficients of the $n$-th rational term for the fuel rod; and $\Sigma_{e}$ is the escape XS of the fuel rod, which is expressed as the inverse of the mean chord length [1].

It should be noted that the subscript $F$ is not indicated in $a_{n}, \beta_{n}$ and $\Sigma_{e}$ for simplification, even though the parameters are for the fuel rod. When the multi-term rational approximation is used, the total flux is fomulated as a linear combination of $n$-th flux. By substituting Eq. (7) into Eq. (5) with the approximation, the flux becomes

$$
\phi_{F}(E)=\sum_{n=1}^{N} \beta_{n} \phi_{F, n}(E)=\sum_{n=1}^{N} \beta_{n} \frac{\lambda_{F} \Sigma_{p, F}+\alpha_{n} \Sigma_{e}}{\Sigma_{a, F}(E)+\lambda_{F} \Sigma_{r s, F}(E)+\lambda_{F} \Sigma_{p, F}+\alpha_{n} \Sigma_{e}} \frac{1}{E},
$$

where $\Sigma_{r s, F}(E)$ is the resonance scattering XS of fuel material.

In the equivalence theory, it is assumed that fuel contains only one resonant nuclide and only the nuclide has an absorption XS. Therefore the $\Sigma_{a, F}(E)$ and $\Sigma_{r s, F}(E)$ are assumed to be both macroscopic XSs of the fuel and the resonant nuclide $r$ (i.e., $\left.\Sigma_{a, F}(E)=N^{r} \sigma_{a}^{r}(E)\right)$. The fuel flux is rewritten as Eq. (9) by dividing the numerator and denominator by number density of target nuclide $r$.

$$
\phi_{F}(E)=\sum_{n=1}^{N} \beta_{n} \phi_{F, n}(E)=\sum_{n=1}^{N} \beta_{n} \frac{\sigma_{b, n}^{r}}{\sigma_{a}^{r}(E)+\lambda^{r} \sigma_{r s}^{r}(E)+\sigma_{b, n}^{r}} \frac{1}{E}
$$

where $\sigma_{a}^{r}(E)$ is the absorption XS of nuclide $r ; \lambda^{r} \sigma_{r s}^{r}(E)$ is the resonance scattering multiplied by the IR parameter of nuclide $r ; \sigma_{b, n}^{r}$ is the $n$-th term background XS of nuclide $r$, which is defined as:

$$
\sigma_{b, n}^{r}=\frac{1}{N^{r}}\left(\lambda_{F} \Sigma_{p, F}+\alpha_{n} \Sigma_{e}\right)
$$

The lethargy form of Eq. (9) is

$$
\phi_{F}(u)=\sum_{n=1}^{N} \beta_{n} \phi_{F, n}(u)=\sum_{n=1}^{N} \beta_{n} \frac{\sigma_{b, n}^{r}}{\sigma_{a}^{r}(u)+\lambda^{r} \sigma_{r s}^{r}(u)+\sigma_{b, n}^{r}} .
$$

Usually, the resonance scattering XS in Eq. (11) is omitted for simplicity. The multi-group 
effective XS is calculated as a ratio of the reaction rate to the flux integrated over the energy range. Therefore, the multi-group XS for reaction $x$ is calculated as

$$
\begin{aligned}
\sigma_{x, g}^{r}= & \frac{\int_{\Delta u_{g}} \sigma_{x}^{r}(u) \phi_{F}(u) d u}{\int_{\Delta u_{g}} \phi_{F}(u) d u}=\frac{\int_{\Delta u_{g}} \sigma_{x}^{r}(u) \sum_{n=1}^{N} \beta_{n} \frac{\sigma_{b, n}}{\sigma_{a}^{r}(u)+\sigma_{b, n}} d u}{\int_{\Delta u_{g}} \sum_{n=1}^{N} \beta_{n} \frac{\sigma_{b, n}}{\sigma_{a}(u)+\sigma_{b, n}} d u} \\
= & \frac{\sum_{n=1}^{N} \beta_{n} \int_{\Delta u_{g}} \sigma_{x}^{r}(u) \frac{\sigma_{b, n}}{\sigma_{a}(u)+\sigma_{b, n}} d u}{\sum_{n=1}^{N} \beta_{n} \int_{\Delta u_{g}} \frac{\sigma_{b, n}}{\sigma_{a}(u)+\sigma_{b, n}} d u}=\frac{\sum_{n=1}^{N} \beta_{n, g} \sigma_{x, n, g}^{r} \phi_{n, g}}{\sum_{n=1}^{N} \beta_{n, g} \phi_{n, g}}
\end{aligned}
$$

where

$$
\begin{aligned}
& \sigma_{x, n, g}^{r}=\sigma_{x, g}^{r}\left(\sigma_{b, n, g}^{r}\right), \quad x=a, s, f, \\
& \phi_{n, g}=\phi_{g}\left(\sigma_{b, n, g}^{r}\right)=\frac{\sigma_{b, n, g}^{r}}{\sigma_{a, n, g}^{r}+\sigma_{b, n, g}^{r}}, \\
& \sigma_{b, n, g}^{r}=\frac{1}{N^{r}}\left(\sum_{r} \lambda_{g}^{r} N^{r} \sigma_{p}^{r}+\alpha_{n, g} \Sigma_{e}\right) .
\end{aligned}
$$

Actually, the IR parameter is energy-dependent because every resonance has a different width. Therefore, energy integrated IR parameter $\lambda_{g}^{r}$ is dependent on energy group as well. The multi-group parameters, such as $\alpha_{n, g}, \beta_{n, g}$ and $\sigma_{b, n, g}^{r}$, also have energy group dependency. Some lattice codes use the resonance integral (RI) form for the effective XS as follows:

$$
\sigma_{x, g}^{r}=\frac{\sum_{n=1}^{N} \beta_{n, g} R I_{x, n, g}^{r}}{1-\sum_{n=1}^{N} \beta_{n, g} \frac{R I_{a, n, g}^{r}}{\sigma_{b, n, g}^{r}}},
$$

where 


$$
R I_{x, n, g}^{r}=\frac{\int_{\Delta u_{g}} \sigma_{x}^{r}(u) \phi_{F, n}(u) d u}{\int_{\Delta u_{g}} d u}=R I_{x, g}^{r}\left(\sigma_{b, n, g}^{r}\right)=\sigma_{x, n, g}^{r} \phi_{n, g}
$$

Eq. (16) is merely a different expression of Eq. (12). The XS and flux are expressed as the multi-group form in Eq. (12), while the reaction rate or RI is written as the multi-group form in Eq. (16). Although the effective XSs in Eq. (12) are derived based on the IR approximation, the effective XS from a slowing-down solution is used in the actual calculation. A table of the effective XSs is pre-generated for various diluted conditions (i.e., background XS). The lattice code calculates a relevant background XS, $\sigma_{b, n, g}^{r}$, for a given problem and then interpolates the effective XS into the pre-generated XS look-up table.

There are various calculation methods for the coefficients of the first flight collision probability in Eq. (7). The enhanced neutron current method [12] solves a fixed source transport equation in Eq. (18) without the resonance scattering XS.

$$
\Omega \cdot \nabla \psi_{g}(v, \Omega)+\left[\Sigma_{a, g}^{*}(v)+\lambda_{g} \Sigma_{p}(v)\right] \psi_{g}(v, \Omega)=\frac{1}{4 \pi} \lambda_{g} \Sigma_{p}(v)
$$

where $\psi_{g}(v, \Omega)$ is the angular flux for the position $v$ and angle $\Omega$; and $\Sigma_{x, g}^{*}(v)$ is the approximated XS of reaction $x$.

In the enhanced neutron current method, the total or absorption XS are assumed to be infinite, and the Dancoff factor is calculated from the total reaction rate of the fuel region. The Dancoff factor can be used in the calculation of the rational approximation with the Wigner's one-term or Carlvik's two-term method [1].

Until now, the conventional equivalence theory has been derived. There are several modified forms of equivalence theory. The WIMS code adds a resonance scattering term in the derivation in Eq. (19) [4]. The resonance scattering part is usually dropped in the conventional theory assuming that the resonance scattering XS does not make a significant contribution. 


$$
\sigma_{x, g}^{r}=\frac{\sum_{n=1}^{N} \beta_{n, g} R I_{x, n, g}^{r}}{1-\sum_{n=1}^{N} \beta_{n, g} \frac{\lambda R I_{r s, n, g}^{r}+R I_{a, n, g}^{r}}{\sigma_{b, n, g}^{r}}} .
$$

Yamamoto proposed the reaction rate preservation method for the multi-term rational approximation [8], focusing on the idea that multi-group flux should have one absorption XS $\tilde{\sigma}_{a, g}^{r}$ in their denominator of all rational terms in Eq. (12), not the $n$-th term dependent absorption XS $\sigma_{a, n, g}^{r}$. The unified absorption XS $\tilde{\sigma}_{a, g}^{r}$ has a relation in Eq. (20). With the new absorption XS, the effective XS is newly defined as Eq. (21)

$$
\begin{aligned}
& \tilde{\sigma}_{a, g}^{r} \sum_{n=1}^{N} \beta_{n, g} \frac{\sigma_{b, n, g}^{r}}{\tilde{\sigma}_{a, g}^{r}+\sigma_{b, n, g}^{r}}=\sum_{n}^{N} \beta_{n} \frac{\sigma_{a, n, g}^{r} \sigma_{b, n, g}^{r}}{\sigma_{a, n, g}^{r}+\sigma_{b, n, g}^{r}}, \\
& \sigma_{x, g}^{r}=\frac{\sum_{n=1}^{N} \beta_{n, g} \sigma_{x, n, g}^{r} \frac{\sigma_{b, n, g}^{r}}{\sigma_{a, n, g}^{r}+\sigma_{b, n, g}^{r}}}{\sum_{n=1}^{N} \beta_{n, g} \frac{\sigma_{b, n, g}^{r}}{\tilde{\sigma}_{a, g}^{r}+\sigma_{b, n, g}^{r}}} .
\end{aligned}
$$

Cao proposed an improved derivation for the effective XS using an integrated flux lookup table similar to the XS look-up table [7]. In the method, the integrated flux is generated as a function of the background XS while the conventional theory uses an asymptotic expression in Eq. (14). With the integrated flux, the effective XS is calculated as follows:

$$
\sigma_{x, g}^{r}=\frac{\sum_{n=1}^{N} \beta_{n, g} \sigma_{x, n, g}^{r} \phi_{n, g}^{S D}}{\sum_{n=1}^{N} \beta_{n, g} \phi_{n, g}^{S D}},
$$

where $\phi_{n, g}^{S D}$ is the integrated flux from the slowing-down solution with $\sigma_{b, n, g}^{r}$ dilution.

\subsection{Numerical Test with Equivalence Theory}

\section{Table 1}


Material composition of base pin-cell problem.

\begin{tabular}{lll}
\hline Material & Nuclide & $\begin{array}{l}\text { Number density } \\
(\# / \text { barn-cm })\end{array}$ \\
\hline Fuel & ${ }^{238} \mathrm{U}$ & $2.22265 \mathrm{E}-02$ \\
\multirow{2}{*}{ Moderator } & ${ }^{16} \mathrm{O}$ & $4.55881 \mathrm{E}-02$ \\
& ${ }^{16} \mathrm{H}$ & $6.67232 \mathrm{E}-02$ \\
& ${ }^{16} \mathrm{O}$ & $3.33616 \mathrm{E}-02$ \\
\hline
\end{tabular}

A base pin-cell problem was designed to demonstrate accuracy of the methods in the previous section. The pin-cell has $0.4 \mathrm{~cm}$ radius of fuel and pin pitch of $1.2 \mathrm{~cm}$. A reflective boundary condition was applied on all sides of the pin-cell. Material compositions are shown in Table 1. The temperature of all regions was 293.6 K. The reference was MCNP6 Monte Carlo code [Error! Reference source not found.]. The numerical test was performed with the lattice physics code STREAM [5]. STREAM uses the method of characteristics (MOC) for transport calculation and the equivalence theory for the resonance treatment. All simulation codes in this paper use ENDF-B/VII.0 nuclear data. A three-term rational approximation was used for the numerical test in this section. Four effective XS calculation methods have been tested.

1) EQ: Conventional equivalence theory with Eq. (16).

2) $\mathrm{EQ} / \mathrm{RR}$ : Equivalence theory with the reaction rate preservation method for multiterm rational approximation in Eq. (21).

3) EQ/RS: Equivalence theory with the resonance scattering part in Eq. (19).

4) EQ/IF: Equivalence theory with integrated flux in Eq. (22). 


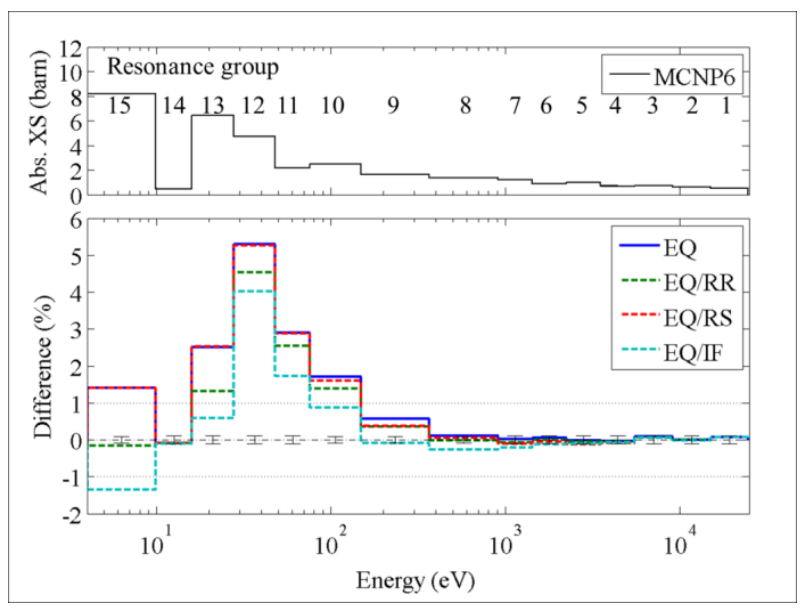

Fig. 1.Comparison of ${ }^{238} \mathrm{U}$ absorption XSs from equivalence theory (base pin-cell problem).

Fig. 1 shows the ${ }^{238} \mathrm{U}$ absorption XS comparison results. The absorption XS of EQ is highly overestimated in resonance groups 9 to 15 . Especially, the absorption XS of group 12 is overestimated by about $5 \%$. EQ shows accurate results in groups 1 to 8 where narrow resonance exists. EQ/RR shows some improvements in computing absorption XS. The absorption XSs in groups 9 to 15 are reduced compared to that of EQ. However, the XSs are still overestimated in groups 9 to 13 . EQ/RS does not have a significant difference from EQ. It means that considering multi-group resonance scattering XS does not have a significant impact on the effective XS calculation. The overestimation of the ${ }^{238} \mathrm{U}$ absorption XS is significantly reduced by using EQ/IF. However, there is still a significant difference in groups 10 to 13 , and the XS of group 15 is overcorrected by the EQ/IF method. The tendency of $\mathrm{EQ} / \mathrm{IF}$ is similar to the original paper [7]. From this numerical test with equivalence theory, it is concluded that the conventional equivalence theory has a significant error in calculating the absorption XS. Several modified methods $[4,7,8]$ show some improvements but a significant error still remains in the XS.

\subsection{Pointwise Energy Approach}

Significant errors were detected in the ${ }^{238} \mathrm{U}$ absorption XS with multi-group approaches (equivalence theory). There are two main reasons of the discrepancy. One is inconsistency between the derivation of the multi-group effective XS and its usage. The other is a flat 
scattering source approximation on the entire fuel region.

The transport equation in Eq. (1) is simplified with the 1/E source approximation on the moderator scattering source and reciprocity relation as follows:

$$
\Sigma_{t, F}(u) \phi_{F}(u)=P_{F F}(u) Q_{s, F}(u)+P_{F M}(u) \Sigma_{t, F}(u)
$$

Eq. (23) is arranged for the flux with the rational approximation in Eq. (7) as follows:

$$
\phi_{F}(u)=\sum_{n=1}^{N} \beta_{n} \frac{Q_{s, F}(u)+\alpha_{n} \Sigma_{e}}{\sum_{t, F}(u)+\alpha_{n} \Sigma_{e}} .
$$

In the derivation of the equivalence theory with the multi-term rational approximation, it is assumed that the total flux is expressed as a linear combination of the $n$-th flux as discussed in Eq. (8). With this assumption and the IR approximation, the flux is written as follows:

$$
\phi_{F}(u)=\sum_{n=1}^{N} \beta_{n} \frac{\lambda \Sigma_{p, F}+\alpha_{n} \Sigma_{e}}{\Sigma_{t, F}(u)+\alpha_{n} \Sigma_{e}}=\sum_{n=1}^{N} \beta_{n} \phi_{F, n}(u)
$$

There is no doubt that the flux can be separated to each $n$-th term because the scattering source of fuel with the IR approximation $\lambda \Sigma_{p, F}$ does not have a dependency on $n$. For the sake of the separation of flux to $n$, the multi-group XS can be written as a function of $n$ (or $n$ th background XS), and the final expression of the effective XS is made as a linear combination of $N$ effective XSs and fluxes.

Although the effective XS is derived based on the IR approximation, the multi-group XS from the slowing-down calculation is used in the practical calculation to achieve a higher accuracy, as discussed in Section 2.1. The multi-group XS is pre-generated as a function of the background XS by solving the slowing down equation, and then the lattice physics code interpolates the multi-group XS using the background XS. The effective XS and background XS have a one-to-one relationship in the look-up table. The discrepancy between derivation of the effective XS and the usage of the XS look-up table is the first reason for the overestimation of the ${ }^{238} \mathrm{U}$ absorption XS. In this process, each $n$-th effective XS $\sigma_{x, n, g}^{r}$ is interpolated with the $n$-th background XS $\sigma_{b, n, g}^{r}$. In other words, the transport equation for the $n$-th system in Eq. (26) is solved independently from other systems. As a result of Eq. 
(26), $\phi_{F, n}(u)$ is known for each $n$-th system. The final effective XS is calculated by using the separated fluxes as Eq. (27).

$$
\begin{aligned}
& \Sigma_{t, F}(u) \phi_{F, n}(u)=\frac{\Sigma_{t, F}(u)}{\sum_{t, F}(u)+\alpha_{n} \Sigma_{e}} Q_{s, F, n}(u)+\left(1-\frac{\Sigma_{t, F}(u)}{\Sigma_{t, F}(u)+\alpha_{n} \Sigma_{e}}\right) \Sigma_{t, F}(u), n=1 \cdots N, \\
& \sigma_{x, g}^{r}=\frac{\int_{\Delta u_{g}} \sigma_{x}^{r}(u) \sum_{n=1}^{N} \beta_{n} \phi_{F, n}(u) d u}{\int_{\Delta u_{g}} \sum_{n=1}^{N} \beta_{n} \phi_{F, n}(u) d u},
\end{aligned}
$$

where $Q_{s, F, n}(u)$ is the fuel scattering source for $n$-th system and defined as

$$
Q_{s, F, n}(E)=\sum_{r \in F} N^{r} \int_{E}^{E / \alpha^{r}} \sigma_{s}^{r}\left(E^{\prime}\right) \phi_{F, n}\left(E^{\prime}\right) \frac{1}{1-\alpha^{r}} \frac{d E^{\prime}}{E^{\prime}}
$$

In Eq. (26), each $n$-th system has its own scattering source and flux. However, the scattering source in Eq. (23) cannot be separated for each $n$-th system when the equation is derived with slowing-down scattering source $Q_{s, F}(u)$ because the total flux, $\phi_{F}$, is positioned in the energy integral and used to calculate the scattering source, as shown in Eq. (2). The scattering source must have information of the entire system. Therefore, the scattering source and the flux should be calculated by solving Eq. (29), and the effective XS by Eq. (30).

$$
\begin{aligned}
& \Sigma_{t, F}(u) \phi_{F}(u)=\sum_{n=1}^{N} \beta_{n} \frac{\Sigma_{t, F}(u)}{\sum_{t, F}(u)+\alpha_{n} \Sigma_{e}} Q_{s, F}(u)+\left(1-\sum_{n=1}^{N} \beta_{n} \frac{\Sigma_{t, F}(u)}{\sum_{t, F}(u)+\alpha_{n} \Sigma_{e}}\right) \Sigma_{t, F}(u), \\
& \sigma_{x, g}^{r}=\frac{\int_{\Delta u_{g}} \sigma_{x}^{r}(u) \phi_{F}(u) d u}{\int_{\Delta u_{g}} \phi_{F}(u) d u} .
\end{aligned}
$$

The discrepancy in the total fluxes calculated from the two ways can be expressed as follows:

$$
\phi_{F}^{S}(u)-\phi_{F}^{C}(u)=\sum_{n=1}^{N} \beta_{n} \frac{Q_{F, n}(u)-Q_{F}(u)}{\sum_{t, F}(u)+\alpha_{n} \Sigma_{e}} \neq 0 .
$$

where $\phi_{F}^{S}(u)$ is the total flux from Eq. (27) by adding all fluxes of separated systems with 
corresponding coefficient $\beta_{n}, \phi_{F}^{C}(u)$ is the flux from Eq. (29).

As shown in Eq. (31), the difference between two types of fluxes is not zero because of the discrepancy in the scattering source. Therefore, it is concluded that the scattering source should not be separated into multiple dilution system.

Unfortunately, there is no way to pre-generate the solution of Eq. (29) for use of the XS look-up table because only one solution (effective XS) of Eqs. (29) and (30) exists while multiple background XSs are used in the equation. The effective XS and the background XS cannot be matched one-to-one.

\subsection{Numerical Test with Pointwise Energy Approach}

The difference in the effective XSs between Eqs. (27) and (30) is numerically tested. A pointwise energy (PW) 0-D slowing-down module was developed to solve the Eq. (29). For this numerical test, a three-term rational approximation is used [5]. The coefficients of the rational equation for same problem in Section 2.2 are shown in Table 2.

Table 2

Coefficients of three-term rational equation

\begin{tabular}{lll}
\hline$n$ & $a_{n}$ & $\beta_{n}$ \\
\hline 1 & 1.422510 & 1.491740 \\
2 & 3.464850 & -0.699455 \\
3 & 5.131570 & 0.207720 \\
\hline
\end{tabular}

In addition to PW 0-D solutions, the identical problem is solved by a PW MOC program. The following are calculation options for this numerical test

1) 1-mesh-PW-MOC: PW MOC solution with 1-mesh flat source region in fuel.

2) 15-mesh-PW-MOC: PW MOC solution with 15-mesh flat source region in fuel.

3) Separated-PW-0D : PW 0-D solution with Eqs. (26) and (27). 
4) Combined-PW-0D: PW 0-D solution with Eqs. (29) and (30).

In the two sets of PW MOC calculations, 1-mesh and 15-mesh flat source regions are used. For comparison of the effective XS of 15-mesh-PW-MOC with MCNP6, the effective $\mathrm{XS}$ of the entire fuel region is compared by taking the average as follows:

$$
\bar{\sigma}_{x, g}^{r}=\frac{\sum_{i \in F} V_{i} \int_{\Delta u_{g}} \sigma_{x}^{r}(u) \phi_{i}(u) d u}{\sum_{i \in F} V_{i} \int_{\Delta u_{g}} \phi_{i}(u) d u},
$$

where $i$ is index of radial submesh of fuel region.

The PW 0-D equations are solved without considering the scattering source or the flux distributions in the fuel region. It is based on the conventional equivalence theory which assumes the one flat source region for the fuel.

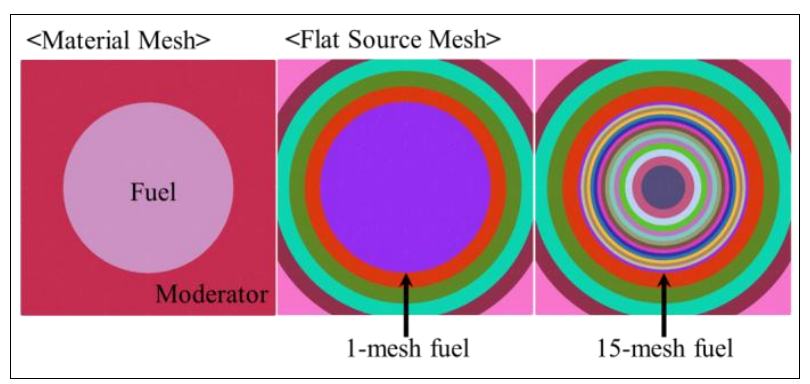

Fig. 2. Flat source mesh divisions of pointwise energy MOC.

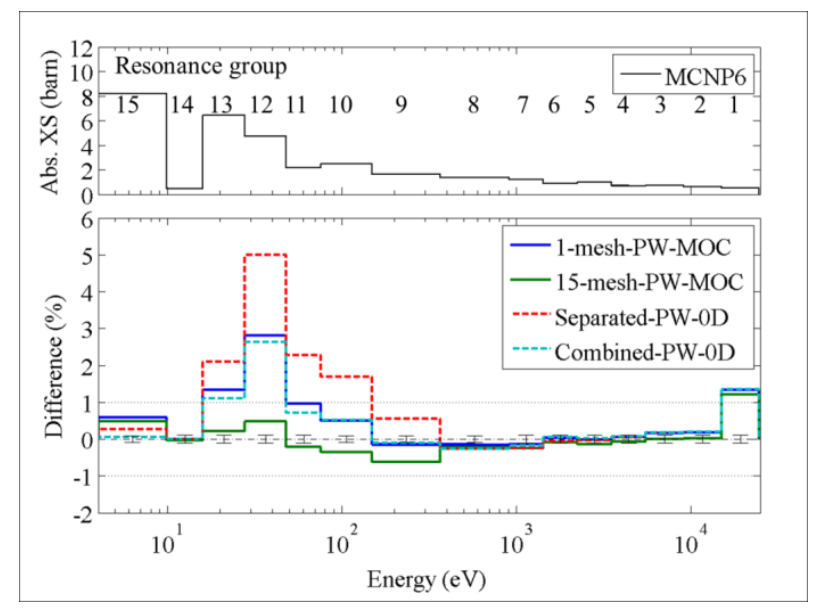

14 / 47 
Fig. 3. Comparison of fuel region averaged ${ }^{238} \mathrm{U}$ absorption XSs from pointwise energy approaches (base pin-cell problem).

Solutions from the methods based on the equivalence theory in Section 2.1 should match with Separated-PW-OD solution if multi-group parameters such as $\lambda_{g}, P_{F F, g}$ and $\sigma_{b, n, g}^{r}$ are properly generated to reproduce the pointwise (or continuous energy) solution. In Fig. 3, Separated-PW-0D shows overestimated ${ }^{238} \mathrm{U}$ absorption XS similarly to the results with equivalence theory in Fig. 1. The absorption XS of group 12 is overestimated by about $5 \%$. Combined-PW-0D shows an improved absorption XS, especially in groups 9 to 13. The maximum difference is less than 3\%. The PW 0-D calculations should reproduce the 1-meshPW-MOC result. The result of Combined-PW-OD is similar to 1-mesh-PW-MOC result. On the other hand, there is a significant difference between Combined-PW-OD and SeparatedPW-0D which comes from whether the scattering source and flux have information on the entire system or independently separated $N$ dilution systems.

Although Combined-PW-0D shows a more accurate solution than Separated-PW-0D, there is still significant error. The remaining error comes from mesh divisions in the flat source regions. This error can be noticed by comparing 1-mesh-PW-MOC and 15-mesh-PWMOC. Most of the error in 1-mesh-PW-MOC disappeared in 15-mesh-PW-MOC. The calculation with one radial mesh of fuel cannot model the distributions of scattering source and flux inside of fuel. However, the distribution of the scattering source inside the fuel is very important in terms of the fuel-to-fuel collision probability and fuel escape probability. The fuel-to-fuel collision probabilities are compared in the Fig. 4. 


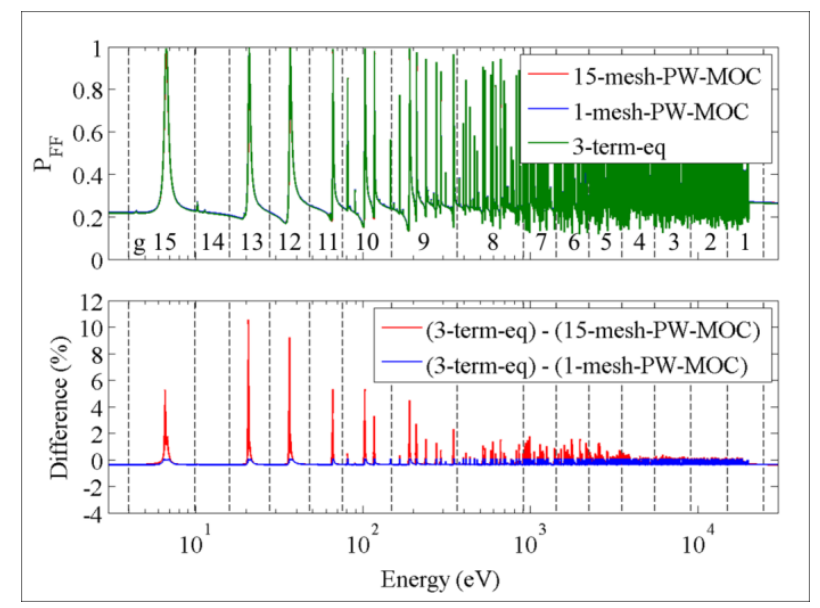

Fig. 4. Comparison of fuel-to-fuel collision probability (base pin-cell problem).

The region averaged fuel-to-fuel collision probabilities of 15-mesh-PW-MOC and 1mesh-PW-MOC are calculated by weighting the scattering source as described in Eq. (33).

$$
P_{F F}(u)=\frac{\sum_{i \in F} \sum_{j \in F} P_{i j}(u) Q_{s, i}(u) V_{i}}{\sum_{i \in F} Q_{s, i}(u) V_{i}},
$$

where $Q_{s, i}(u)$ is the scattering source of submesh $i$; and $P_{i j}(u)$ is first flight collision probability from $i$ to $j$.

The way to calculate the collision probability $P_{i j}(u)$ is described in Section 3.2. 3-termeq represents the three-term rational approximation in Eq. (7)

$$
P_{F F}(u)=\sum_{n=1}^{N} \frac{\beta_{n} \Sigma_{t, F}(u)}{\Sigma_{t, F}(u)+\alpha_{n} \Sigma_{e}}
$$

As shown in Fig. 4, 3-term-eq and 1-mesh-PW-MOC show quite similar $P_{F F}$, with less than $0.36 \%$ difference. However, there is quite a significant difference between 3-term-eq and 15 -mesh-PW-MOC in groups 9 to 15 . Especially, about $10 \%$ difference is detected in groups 12 and 13. This discrepancy occurs because the scattering source distribution inside the fuel is not modeled in 1-mesh-PW-MOC and 3-term-eq. ${ }^{238} \mathrm{U}$ has large resonance scattering XSs in $15 \mathrm{eV} \sim 75 \mathrm{eV}$. Neutrons that travel from a moderator into a fuel has scattering and absorption reactions in the peripheral area of the fuel. Thus, the flux is 
dramatically depressed from the outer to inner region of the fuel near the resonance peak energy. The scattering source distribution also shows a profound depression from surface to the inner part of the fuel as shown in Fig. 5. The large resonance scattering XS of ${ }^{238} \mathrm{U}$ makes this phenomena more significant. Definitely, neutrons in the outer region escape from the fuel more easily. That is why the fuel-to-fuel collision probabilities of 15-mesh-PW-MOC always are smaller than those of 1-mesh-PW-MOC and 3-term-eq. In the resonance treatment, the discrepancy in the fuel-to-fuel collision probability causes the error in the effective XS.

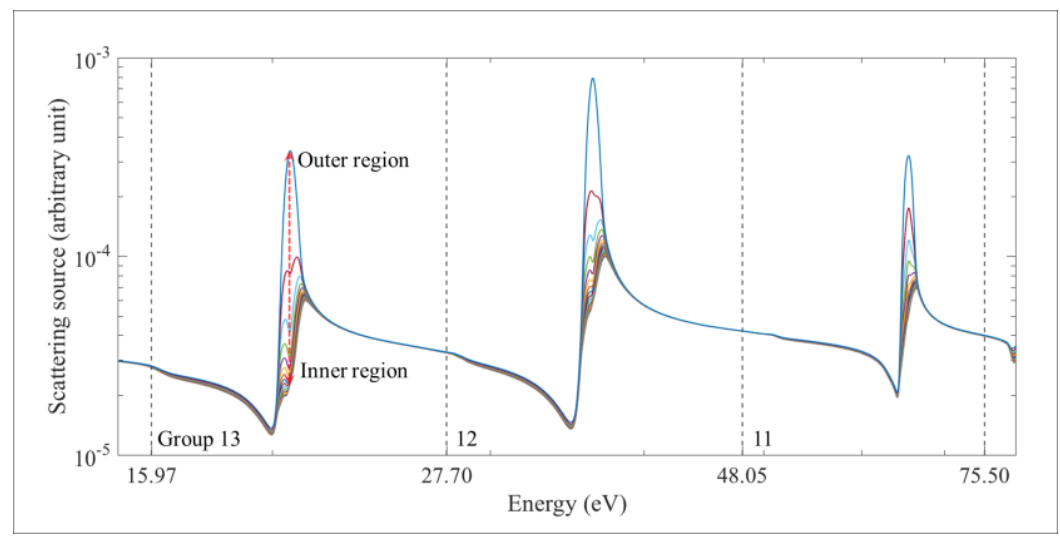

Fig. 5. Scattering source distribution in fuel pellet from 15-mesh-PW-MOC.

\subsection{Numerical Test without Resonance Scattering Cross Section}

In Section 2.4, it was noted that the use of XS look-up table and the scattering source distribution inside the fuel are the main reason for the overestimation of multi-group effective $\mathrm{XS}$ in the equivalence theory. However, the equivalence theory even with the two problems has been used in many lattice codes, giving a reasonable solution. In this section, the point at which the equivalence theory causes the biased solution will be discussed.

This work focuses on the resonance scattering XS of ${ }^{238} \mathrm{U} \cdot{ }^{238} \mathrm{U}$ has a very large and wide resonance scattering XSs in the epithermal energy. The resonance scattering XSs cause a significant error in the solution of the equivalence theory. In Fig. 6, the total, absorption, and scattering XSs of ${ }^{238} \mathrm{U}$ are shown. A fictitious ${ }^{238} \mathrm{U}$ is generated in order to verify that the resonance scattering causes the bias. The fictitious ${ }^{238} \mathrm{U}$ contains only the potential XS in the scattering XS. 


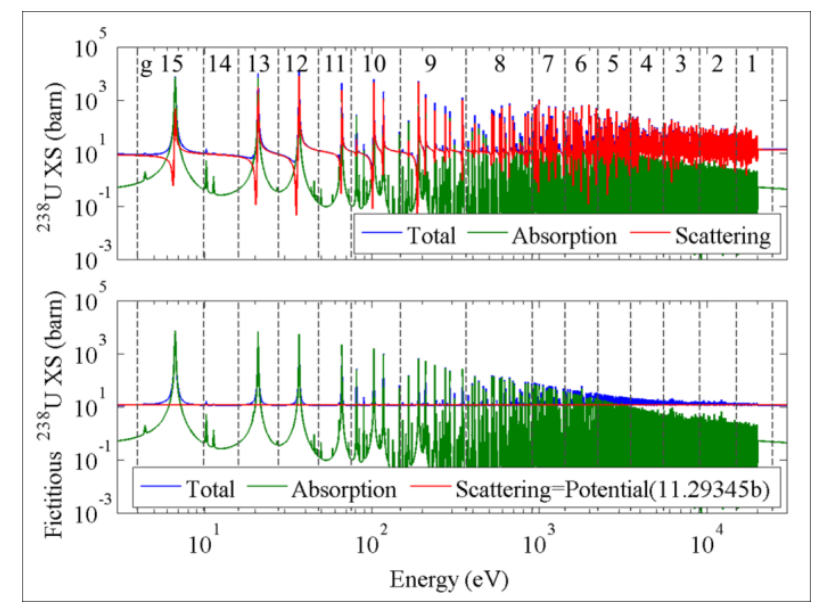

Fig. 6. Cross Sections of ${ }^{238} U$ and fictitious ${ }^{238} U$.

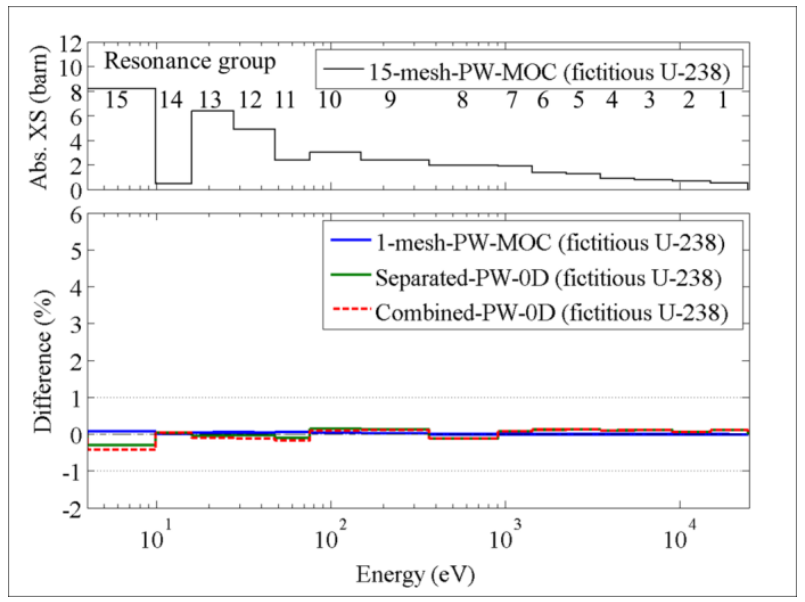

Fig. 7. Comparison of fictitious ${ }^{238} \mathrm{U}$ absorption XSs from pointwise energy approaches (base pin-cell problem).

The identical problem in Section 2.2 is solved by four different methods (1-mesh-PWMOC, 1-mesh-PW-MOC, Separated-PW-0D and Combined-PW-0D). In the four methods, a modified PW XS library is used in order to model the fictitious ${ }^{238} \mathrm{U}$. In this comparison, 15mesh-PW-MOC is set as a reference for the verification purpose of the bias effect. As shown in Fig. 7, the result is quite different from Section 2.4. All four methods show quite similar results each other. 1-mesh-PW-MOC has less than $0.1 \%$ difference, compared to 15-mesh- 
PW-MOC. It indicates that the distribution of the scattering source does not have a significant impact on computing the effective XS for the fictitious ${ }^{238} \mathrm{U}$. The amount of scattering source of the fictitious ${ }^{238} \mathrm{U}$ is very small compared to the source from the moderator so that the distribution of scattering source of fuel is meaningless. It was discussed in Section 2.4 that the effective XS from the equivalence theory should be identical to that of Separated-PW-OD, if the multi-group parameters are generated properly to reproduce the pointwise energy solution. In Section 2.4, a significant difference was detected in the effective XS from Separated-PW-0D and Combined-PW-0D, because the scattering source of Separated-PW-0D is divided into $N$ systems and calculated independently. However, if ${ }^{238} \mathrm{U}$ does not have a resonance scattering XS, the difference between the solutions from Separated-PW-OD and Combied-PW-0D disappears. This is because the scattering source is also very small compared to the source from moderator. For the case that the resonance scattering XS is not large, the equivalence theory can produce a reasonable solution.

\section{New Resonance Self-Shielding Method}

In Section 2, the limitations of the equivalence theory are discussed. There is an error in the derivation of equivalence theory with the multi-term rational approximation. Furthermore, one region assumption inside the fuel, which is typically used in the equivalence theory, causes a significant error in the final effective XS. Therefore, a new resonance self-shielding method is required to correct the errors in the equivalence theory. In Section 3.1, the limitations of existing spatially dependent self-shielding methods are discussed. In Section 3.2, a new resonance self-shielding method is proposed based on the pointwise energy slowing-down solution. Section 3.3 describes the calculation flow of the method.

\subsection{Contemporary Spatially Dependent Self-Shielding Method}

There are several existing methods for spatial self-shielding inside a fuel rod with the equivalence theory. CASMO-5 [10] calculates the averaged effective XS of the entire fuel rod, and then applies an empirical radial distribution function, which is generated from a MCNP calculation, for the ${ }^{238} \mathrm{U}$ resonance integral. Matsumoto developed the spatially dependent Dancoff method (SDDM) [11] based on the idea of Stoker-Weiss [9]. SDDM calculates the 
coefficients of the rational approximation using the Dancoff factor for the entire fuel rod, and applies a weighting function for a spatial self-shielding inside the fuel rod. Both the CASMO5 method and the SDDM can successfully consider the radial self-shielding effect inside the fuel pellet. However, both methods have the following two drawbacks. First, the methods use multi-term rational approximation (Carlvik's two-term) and the effective XS comes from the XS look-up table using multiple background XSs. The underlying error in this process is discussed in Sections 2.3 and 2.4. Second, both methods use resonance parameters or an effective XS calculated for an averaged fuel rod. CASMO-5 normalizes the corrected effective XS as follows:

$$
\sum_{i \in F} N_{i}^{\mathrm{U} 238} V_{i} \sigma_{a, i, g}^{\mathrm{U} 238}=\sum_{i \in F} N_{i}^{\mathrm{U} 238} V_{i} \bar{\sigma}_{a, g}^{\mathrm{U} 238}
$$

where $N_{i}^{\mathrm{U} 238}$ is the number density of ${ }^{238} \mathrm{U}$ in submesh $i ; \bar{\sigma}_{a, g}^{\mathrm{U} 238}$ is the averaged absorption $\mathrm{XS}$ of ${ }^{238} \mathrm{U} ; \sigma_{a, i, g}^{\mathrm{U} 238}$ is the corrected absorption XS of ${ }^{238} \mathrm{U}$ in submesh $i$ with the empirical correction factor $w_{i}$. as

$$
\sigma_{a, i, g}^{\mathrm{U} 238}=w_{i} \bar{\sigma}_{a, g}^{\mathrm{U} 238}
$$

If the averaged ${ }^{238} \mathrm{U}$ absorption XS is not calculated properly (as discussed in Section 2.4), the distributed absorption XS still has bias in the averaged XS. SDDM calculates the effective XS of submesh $i$ using the coefficients of the rational approximation for the averaged fuel as follows:

$$
\sigma_{x, i, g}^{r}=\frac{\sum_{m=1}^{4} F_{i, m} \sum_{n=1}^{N} \beta_{n, g} R I_{x, g}^{r}\left(\sigma_{b, i, n, m, g}^{r}\right)}{1-\sum_{m=1}^{4} F_{i, m} \sum_{n=1}^{N} \beta_{n, g} \frac{R I_{a, g}^{r}\left(\sigma_{b, i, n, m, g}^{r}\right)}{\sigma_{b, i, n, m, g}^{r}}},
$$

where $F_{i, m}$ is the weighting function; and $\sigma_{b, i, n, m, g}^{r}$ is the background XS defined as

$$
\sigma_{b, i, n, m, g}^{r}=\frac{1}{N^{r}}\left(\sum_{r} \lambda_{g}^{r} N^{r} \sigma_{p}^{r}+\alpha_{n, g} \Sigma_{e, i, m}\right),
$$

where $\Sigma_{e, i, m}$ is escape XS of submesh $i$ and shape $m$. 
The fuel-to-fuel collision probability generated for the averaged fuel rod has a significant error because of the scattering source distribution inside of a fuel is not modeled as shown in Fig. 4. Therefore, SDDM also has the same problem as long as $\alpha_{n, g}$ and $\beta_{n, g}$ are calculated for the fuel lump.

Liu developed a quasi-one-dimensional slowing-down method to account for the spatially dependent resonance self-shielding effect [14]. A correction factor is generated from the continuous energy slowing-down solution and then multiplied to the multi-group XS which is generated by using the embedded self-shielding method (ESSM). The quasi-1D method with ESSM shows good accuracy in calculating reactor parameters. However, the quasi-1D method cannot be used in correcting the error from the resonance scattering source discussed in Section 2. In the quasi-1D method, the continuous energy slowing-down solution is used in generating the XS correction factor as follows:

$$
\sigma_{x, i, g}^{r, \text { Final }}=\sigma_{x, F, g}^{r, \text { ESSM }} f_{x, i, g}^{r}=\sigma_{x, F, g}^{r, \text { ESSM }} \frac{\tilde{\sigma}_{x, i, g}^{r \text {,inf }}}{\tilde{\sigma}_{x, F, g}^{r, \text { non-inf }}}
$$

where $\sigma_{x, F, g}^{r \text {,ESM }}$ is the fuel region averaged multi-group XS of nuclide $r$ from ESSM; $\tilde{\sigma}_{x, i, g}^{r \text {,intf }}$ is the spatially dependent multi-group XS nuclide $r$ from quasi-1D slowing-down solution; and $\tilde{\sigma}_{x, F, g}^{r, \text { non-inff }}$ is the fuel region averaged multi-group XS nuclide $r$ from quasi-1D slowing-down solution.

Both XSs, $\tilde{\sigma}_{x, i, g}^{r \text {,inff }}$ and $\tilde{\sigma}_{x, F, g}^{r, \text { non-inff }}$ in Eq. (38), are generated from a heterogeneous system (quasi-1D). Moreover, the base multi-group XS $\sigma_{x, F, g}^{r, \text { ESSM }}$ also comes from the heterogeneous RI table [15]. ESSM uses the heterogeneous RI table, which is generated by solving a continuous energy slowing-down problem with 2-D pin-cell transport solver. The resonance scattering source distribution is spontaneously considered in the RI generation process so that ESSM does not have bias from the resonance scattering source. In other words, ${ }^{238} \mathrm{U}$ effective XS from ESSM without the quasi-1D method does not have an error from the resonance scattering source. However, the generation of the heterogeneous RI table is much more difficult than the conventional XS generation process as a significant effort is required to generate the heterogeneous RI table as a function of background XS and fuel to moderator volume ratio. 


\subsection{Pin-based Pointwise Energy Slowing-Down Method}

In order to resolve drawbacks in the contemporary equivalence theory, a pin-based pointwise energy slowing-down method (PSM) is proposed in this paper. The transport equation in Eq. (1) is re-written with radially divided submeshes [9] as follows:

$$
\Sigma_{t, i}(E) \phi_{i}(E) V_{i}=\sum_{j \in F} P_{j i}(E) V_{j} Q_{s, j}(E)+P_{M i}(E) V_{M} Q_{s, M}(E)
$$

where the subscripts $i$ and $j$ are indexes of radial submesh of the fuel region; and the slowing-down scattering source of submesh $j$ is defined as

$$
Q_{s, j}(E)=\sum_{r \in j} N^{r} \int_{E}^{E / \alpha^{r}} \sigma_{s}^{r}\left(E^{\prime}\right) \phi_{j}\left(E^{\prime}\right) \frac{1}{1-\alpha^{r}} \frac{d E^{\prime}}{E^{\prime}}
$$

Using a reciprocity relation, Eq. (39) is written with a lethargy form as follows:

$$
\Sigma_{t, i}(u) \phi_{i}(u)=\sum_{j \in F} P_{i j}(u) \frac{\sum_{t, i}(u)}{\sum_{t, j}(u)} Q_{s, j}(u)+P_{i M}(u) \frac{\sum_{t, i}(u)}{\sum_{p, M}} Q_{s, M}(u)
$$

where the reciprocity relation is

$$
\left\{\begin{array}{l}
P_{j i}(u) \Sigma_{t, j}(u) V_{j}=P_{i j}(u) \Sigma_{t, i}(u) V_{i} \\
P_{M i}(u) \Sigma_{p, M} V_{M}=P_{i M}(u) \Sigma_{t, i}(u) V_{i}
\end{array} .\right.
$$

In Eq. (41), the index $M$ indicates the non-fuel region. In case of the multi-region problem (i.e., fuel/gap/clad/moderator), non-fuel regions are merged with volume weighting by assuming a constant spatial flux in non-fuel regions. The non-fuel regions of each unit pincell is merged into one region. In addition, it is assumed that non-fuel material only has a potential XS. By rearranging Eq. (41), the expression for the flux of region $i$ is written as

$$
\phi_{i}(u)=\sum_{j \in F} \frac{P_{i j}(u)}{\sum_{t, j}(u)} Q_{s, j}(u)+\frac{P_{i M}(u)}{\sum_{p, M}} Q_{s, M}(u)
$$

Similarly with Eq. (39), the transport equation for the non-fuel region is written as follows: 


$$
\Sigma_{p, M} \phi_{M}(u) V_{M}=\sum_{i \in F} P_{i M}(u) V_{i} Q_{s, i}(u)+P_{M M}(u) V_{M} Q_{s, M}(u)
$$

where $P_{M M}(u)$ is the non-fuel to non-fuel collision probability. Eq. (44) is arranged to the non-fuel flux as follows:

$$
\phi_{M}(u)=\sum_{i \in F} \frac{P_{M i}(u)}{\sum_{t, i}(u)} Q_{s, i}(u)+\frac{P_{M M}(u)}{\sum_{p, M}} Q_{s, M}(u)
$$

If $P_{i j}(u), P_{i M}(u), P_{M i}(u)$ and $P_{M M}(u)$ are known, the fluxes and scattering sources in Eq. (43) and Eq. (45) can be calculated with the fixed source at high energy. Eq. (43) and Eq. (45) should be calculated simultaneously because the equations are coupled with the collision probability.

The way to calculate collision probabilities is based on the optimum rational approximation method in the STREAM code [5]. In the optimum rational approximation method, the collision probability of an isolated fuel pin is calculated as a function of the wide range of the total XS of fuel (i.e., $10^{-3}$ to $10^{4} \mathrm{~cm}^{-1}$ ) by using the MOC transport solver. The multi-term rational equation is generated through a least square fitting process into the calculated collision probability versus the total XS table. The effects of the neighboring fuel rod or structure material (so called "shadowing effect") are considered by multiplying XS correction factors. The XS correction factor is defined as a ratio of XSs of fuel pin in actual problem to XS of fuel in infinite lattice for capture, scattering and fission reactions. In the optimum rational approximation method, the fuel pellet is assumed to be a single region. Y. Liu used a similar concept in computing collision probabilities with consideration of intrapellet distribution in the quasi-1-D slowing-down method [14].

In PSM, the similar approaches to those used in the optimum rational approximation method and the quasi-1-D method are used in order to calculate $P_{i j}(u)$ efficiently. The collision probability versus total XS table is calculated for an isolated pin with a void boundary condition, and the shadowing effect is considered by the collision probability using the Carlvik's two-term rational approximation. When the collision probability from neutrons born in fuel submesh $i$, the transport equation in Eq. (46) is solved with a unity fixed source at the region of interest (submesh $i$ ). 


$$
\Omega \cdot \nabla \psi(v, \Omega)+\Sigma_{t}^{*}(v) \psi(v, \Omega)=\frac{1}{4 \pi} \delta_{i}(v),
$$

where $v$ is the position; $\Sigma_{t}^{*}(v)$ is a level of total XSs; and $\delta_{i}(v)$ is defined as

$$
\left\{\begin{array}{l}
\delta_{i}(v)=1 \text { if } v \in i \\
\delta_{i}(v)=0 \text { if } v \notin i
\end{array} .\right.
$$

The first flight collision probability from $i$ to $j$ for an isolated fuel pin is calculated by Eq. (48).

$$
P_{i j}^{i s o}\left(\Sigma_{t, i}^{*}\right)=\frac{\Sigma_{t, j}^{*} \phi_{j} V_{j}}{V_{i}}
$$

The superscript $*$ denotes an estimated value, not an actual total XS at specific energy. It should be noted that constant total XS is assumed for the entire fuel submesh for one fixed source calculation although the total XS in Eq. (48) has subscripts $i$ and $j . P_{i j}^{i s o}\left(\Sigma_{t, i}^{*}\right)$ is calculated and saved as a table for a wide range of total XS (i.e., 200 levels from $10^{-3}$ to $10^{4}$ $\left.\mathrm{cm}^{-1}\right)$ of fuel and all submeshes of each pin-type. $P_{i j}^{i s o}\left(\Sigma_{t, i}^{*}\right)$ depends on geometry only so that the calculation is performed for a pin-geometry type, not all fuel pins in a given problem. Fig. 8 shows an example of $P_{i j}^{i s o}\left(\Sigma_{t, i}^{*}\right)$ which is the collision probability of a neutron born in submesh 3 and that had a first collision in submesh $j$. The computation of $P_{i j}^{i s o}\left(\Sigma_{t, i}^{*}\right)$ is not expensive compared to a MOC assembly calculation and thus the computing time is negligible. In case of a cylindrical fuel rod, the collision probability method (CPM) can be applied for faster computation [15]. 


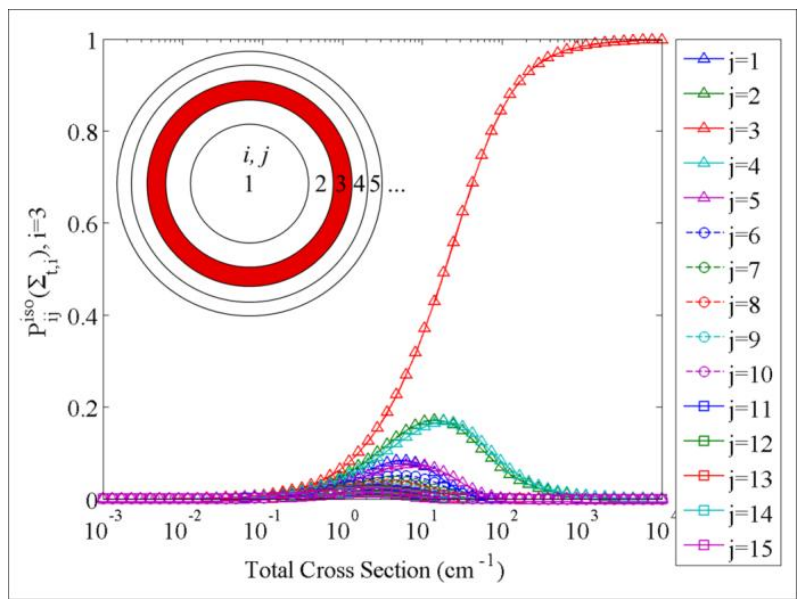

Fig. 8. Example for fuel collision probability of neutron born in submesh 3.

Since Eq. (48) involves a collision probability of an isolated fuel rod, a proper correction on the shadowing effect is required in order to consider the shadowing effect among fuel rods and structure material. The shadowing effect is accounted for using the fuel rod averaged escape probability. The Carlvik's two-term rational approximation can be applied in case of a cylindrical rod as follows:

$$
\begin{aligned}
& \bar{P}_{e, F}^{i s o}\left(\Sigma_{t, F}^{*}\right)=1-\bar{P}_{F F}^{i s o}\left(\Sigma_{t, F}^{*}\right)=2 \frac{2 \Sigma_{e}}{\Sigma_{t, F}^{*}+2 \Sigma_{e}}-\frac{3 \Sigma_{e}}{\Sigma_{t, F}^{*}+3 \Sigma_{e}}, \\
& \bar{P}_{e, F}\left(\Sigma_{t, F}^{*}\right)=1-\bar{P}_{F F}\left(\Sigma_{t, F}^{*}\right)=\beta_{1} \frac{\alpha_{1} \Sigma_{e}}{\Sigma_{t, F}^{*}+\alpha_{1} \Sigma_{e}}+\beta_{2} \frac{\alpha_{2} \Sigma_{e}}{\sum_{t, F}^{*}+\alpha_{2} \Sigma_{e}},
\end{aligned}
$$

where $\bar{P}_{e}^{i s o}\left(\Sigma_{t, F}^{*}\right)$ is the fuel escape probability of an isolated fuel rod; $\bar{P}_{e}\left(\Sigma_{t, F}^{*}\right)$ is the fuel escape probability of fuel rod in lattice; bar indicates a parameter generated with one flat source region assumption inside fuel pellet; and the coefficients in Eq. (50) are defined as follows:

$$
\left\{\begin{array}{l}
A=\Gamma /(1-\Gamma) \\
\alpha_{1,2}=\left(5 A+6 \mp \sqrt{A^{2}+36 A+36}\right) /(2 A+2) \\
\beta_{1}=\left(\frac{4 A+6}{A+1}-\alpha_{1}\right) /\left(\alpha_{2}-\alpha_{1}\right) \\
\beta_{2}=1-\beta_{1}
\end{array},\right.
$$


where $\Gamma$ is the Dancoff factor.

The Dancoff factor is calculated using the enhanced neutron current method [12] by solving the following MOC fixed source problem for a whole problem.

$$
\Omega \cdot \nabla \psi(v, \Omega)+\left(\Sigma_{a}^{*}(v)+\Sigma_{p}(v)\right) \psi(v, \Omega)=\frac{1}{4 \pi} \Sigma_{p}(v),
$$

where $\Sigma_{a}^{*}(v)$ is the estimated absorption XS of each region; and $\Sigma_{p}(v)$ is the macroscopic potential XS of each region.

In the whole-problem MOC fixed source calculation, the absorption XSs are set to be of an infinite value (i.e., $10^{5} \mathrm{~cm}^{-1}$ ) for the fuel region and zero for the non-fuel region. The Dancoff factor is calculated for an individual fuel pin with the fuel total reaction rate, the potential XS and the escape XS of fuel as Eq. (53).

$$
\Gamma=\frac{\left(\Sigma_{a, F}^{*}+\Sigma_{p, F}\right) \phi_{t, F}-\Sigma_{p, F}}{\Sigma_{e}}
$$

It should be noted that Eqs. (52) and (53) do not have IR parameters because the parameters from the fixed source calculation will be used in the pointwise slowing-down calculation. Because the potential XS is independent to energy, the Dancoff factor calculation is performed only one time for the fuel.

The shadowing effect correction factor, which adjusts the fuel escape probability of isolated fuel pin to consider the shadowing effect, is defined as a ratio of fuel escape probabilities of two systems as follows:

$$
\eta_{F}\left(\Sigma_{t, F}^{*}\right)=\frac{\bar{P}_{e, F}\left(\Sigma_{t, F}^{*}\right)}{\bar{P}_{e, F}^{i s}\left(\Sigma_{t, F}^{*}\right)} .
$$

Fig. 9 shows an example of the shadowing effect correction factor. The escape probability becomes unity when the fuel rod is white while it goes to zero when the fuel becomes a black pin. In the black pin limit, the correction factor is identical to the Dancoff factor, which is a ratio of the fuel escape probability of a pin in a lattice to that of the isolated pin with an infinite total XS of fuel. 


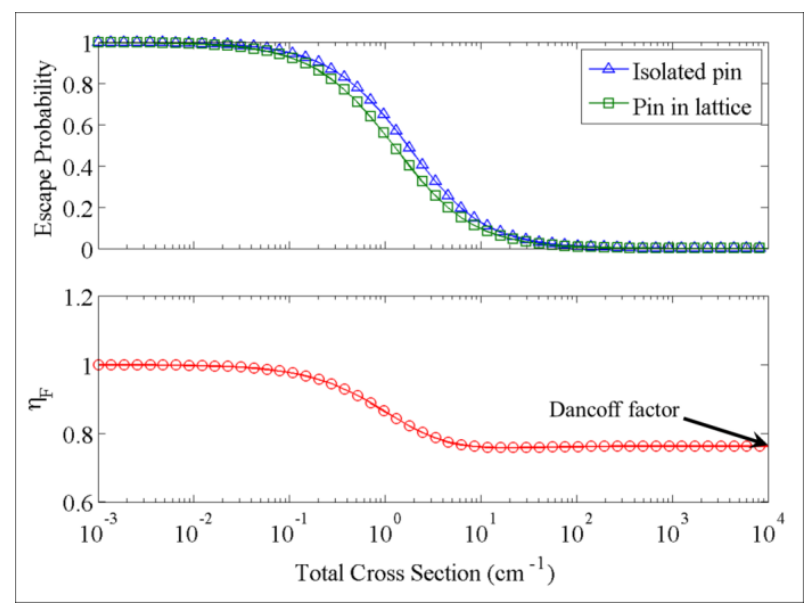

Fig. 9. Example for fuel escape probability of fuel lump and ratio.

The shadowing effect correction factor is multiplied to the fuel escape probability of each submesh of fuel rod as shown in Eq. (55).

$$
P_{i M}\left(\Sigma_{t, i}^{*}\right)=P_{e, i}\left(\Sigma_{t, i}^{*}\right)=\eta_{i}\left(\Sigma_{t, i}^{*}\right) P_{e, i}^{i s o}\left(\Sigma_{t, i}^{*}\right)
$$

where

$$
\begin{aligned}
& P_{i M}^{i s o}\left(\Sigma_{t, i}^{*}\right)=P_{e, i}^{i s o}\left(\Sigma_{t, i}^{*}\right)=1-\sum_{j \in F} P_{i j}^{i s o}\left(\Sigma_{t, i}^{*}\right), \\
& \eta_{i}\left(\Sigma_{t, i}^{*}\right) \approx \eta_{F}\left(\Sigma_{t, i}^{*}\right) .
\end{aligned}
$$

It is assumed that the shadowing effect correction factor of submesh $i$ is equal to that of the entire fuel as shown in Eq. (57). As discussed in Section 2.4, the fuel escape probability may have an error because the escape probability is calculated for a single mesh of the fuel rod without modelling of the scattering source distribution inside of fuel. However, the error does not appear because the correction factor is defined as a ratio of two escape probabilities so that errors included in both probabilities are canceled out. The fuel-to-fuel collision probability is normalized in order to consider the changes in the fuel escape probability as follows:

$$
P_{i j}\left(\Sigma_{t, i}^{*}\right)=P_{i j}^{i s o}\left(\Sigma_{t, i}^{*}\right) \frac{1-P_{e, i}\left(\Sigma_{t, i}^{*}\right)}{1-P_{e, i}^{i s o}\left(\Sigma_{t, i}^{*}\right)} .
$$


The collision probabilities from the non-fuel region can be expressed as follows:

$$
\begin{aligned}
& P_{M i}\left(\Sigma_{t, i}^{*}\right)=\frac{P_{i M}\left(\Sigma_{t, i}^{*}\right) \Sigma_{t, i}^{*} V_{i}}{\sum_{p, M} V_{M}}, \\
& P_{M M}\left(\Sigma_{t, i}^{*}\right)=1-\sum_{i \in F} P_{M i}\left(\Sigma_{t, i}^{*}\right)=1-\sum_{i \in F} \frac{P_{i M}\left(\Sigma_{t, i}^{*}\right) \Sigma_{t, i}^{*} V_{i}}{\sum_{p, M} V_{M}} .
\end{aligned}
$$

Finally, all of the collision probabilities are derived as a function of the total XSs. The flux of submesh $i$ of the fuel rod and the flux of the non-fuel region are written as follows:

$$
\begin{aligned}
& \phi_{i}(u)=\sum_{j \in F} \frac{P_{i j}\left(\Sigma_{t, i}(u)\right)}{\sum_{t, j}(u)} Q_{s, j}(u)+\frac{P_{i M}\left(\Sigma_{t, i}(u)\right)}{\Sigma_{p, M}} Q_{s, M}(u), \quad i \in F, \\
& \phi_{M}(u)=\sum_{i \in F} \frac{P_{M i}\left(\Sigma_{t, i}(u)\right)}{\sum_{t, i}(u)} Q_{s, i}(u)+\frac{P_{M M}\left(\Sigma_{t, i}(u)\right)}{\sum_{p, M}} Q_{s, M}(u) .
\end{aligned}
$$

In Eqs. (61) and (62), $\Sigma_{t, i}^{*}$ is replaced with $\Sigma_{t, i}(u)$ to indicate the total XS corresponding energy $u$. Because the collision probability is a function of the total XS, a simple interpolation into the look-up table allows to easily calculate the collision probabilities at any energy of interest. In actual calculation, only $P_{i j}\left(\Sigma_{t, i}^{*}\right)$ is tabulated as a look-up table and $P_{i j}\left(\Sigma_{t, i}(u)\right)$ is interpolated using the total XS. The remaining collision probabilities are calculated using $P_{i j}\left(\Sigma_{t, i}(u)\right)$. Eqs. (61) and (62) are solved simultaneously to calculate the scattering source. There are several techniques which calculate the scattering source efficiently in order to avoid a time consuming direct integration [17]. If the pointwise energy fluxes of every submesh is calculated, XSs of nuclides in the fuel material are condensed into a multi-group XS as

$$
\sigma_{x, i, g}^{r}=\frac{\int_{\Delta u_{g}} \sigma_{x}^{r}(u) \phi_{i}(u) d u}{\int_{\Delta u_{g}} \phi_{i}(u) d u} .
$$

The resonance interference effect is considered spontaneously in $\sigma_{x, i, g}^{r}$ because mixture of nuclides in fuel is considered during the solution of the pointwise energy slowing-down problem. 
The pointwise slowing-down calculation is performed with an asymptotic elastic scattering kernel. It has been reported that the asymptotic elastic scattering kernel can lead to a $10 \%$ difference in the Doppler coefficient compared to that of a correct scattering model, which considers an exact resonance upscattering event in the epithermal energy range [18]. However, it is hard to solve the pointwise slowing-down equation with the exact scattering kernel on-the-fly. Therefore, the effect of a resonance upscattering event is corrected in the multi-group level using pre-generated upscattering correction factor generated by a Monte Carlo simulation. Nowadays, the resonance upscattering effect is a well-known issue and proper correction methods have been implemented in many transport codes $[5,15,18,19]$. The resonance upscattering correction can be applied to PSM in the similar manner. The upscattering correction factor is defined as a ratio of a ${ }^{238} \mathrm{U}$ multi-group XS from an exact scattering kernel to that from an asymptotic scattering kernel as follows:

$$
f_{x, g}^{R U P}\left(\sigma_{b}^{\mathrm{U}-238}\right)=\frac{\sigma_{x, g}^{R U P}\left(\sigma_{b}^{\mathrm{U}-238}\right)}{\sigma_{x, g}^{A s y .}\left(\sigma_{b}^{\mathrm{U}-238}\right)},
$$

where $x$ is the reaction type; and $\sigma_{b}^{\mathrm{U}-238}$ is the background XS of ${ }^{238} \mathrm{U}$.

Through a table look-up with a background XS versus a multi-group XS look-up table, the equivalent background XS of ${ }^{238} \mathrm{U}, \sigma_{b, e q}^{\mathrm{U} 238}$, corresponding to $\sigma_{x, i, g}^{\mathrm{U}-238}$ is calculated. Using the equivalent background $\mathrm{XS}$, the resonance upscattering effect is corrected as follows:

$$
\tilde{\sigma}_{x, i, g}^{\mathrm{U}-238}=\sigma_{x, i, g}^{\mathrm{U}-238} f_{x, g}^{R U P}\left(\sigma_{b, e q}^{\mathrm{U}-238}\right)
$$

where $\sigma_{x, i, g}^{\mathrm{U}-238}$ is the ${ }^{238} \mathrm{U}$ effective $\mathrm{XS}$ from slowing-down calculation; $\tilde{\sigma}_{x, i, g}^{\mathrm{U}-238}$ is the upscattering corrected effective XS.

The upscattering correction is performed for ${ }^{238} \mathrm{U}$ only. The correction can be used as an alternative way instead of solving the exact scattering kernel on-the-fly.

\subsection{Calculation Flow of Pin-based Pointwise Energy Slowing-Down Method}

The flowchart of PSM is shown in Fig. 10. The calculation process is as follows: 
1) Prepare a 72 group multi-group XS library and a $5 \cdot 10^{4}$ equal lethargy pointwise energy XS library.

2) Read input information and the multi-group XS library.

3) Generate the $P_{i j}^{i s o}$ vs. total XS table for isolated fuel pin using MOC fixed source solver or CPM and Eq.(48). Construct the table for all fuel pin geometry types.

4) Calculate the individual Dancoff factor of fuel pins using Eq. (53) through the whole-problem MOC fixed source calculation.

5) Calculate $\bar{P}_{e, F}^{i s o}$ and $\bar{P}_{e, F}$ by using Eqs. (49) and (50), and then calculate the shadowing effect correction factor $\eta_{F}$ by using Eq. (54).

6) Correct the shadowing effect using the shadowing effect correction factor as Eq. (55), and then calculate $P_{i j}, P_{i M}, P_{M i}$ and $P_{M M}$ with Eqs. (55) through (60).

7) Calculate pointwise energy fluxes by solving spatially dependent slowing-down equations in Eqs. (61) and (62).

8) Condense the pointwise XS to position-dependent multi-group XS. Consider the resonance upscattering effect by Eq. (65) for ${ }^{238} \mathrm{U}$ only. Return to Step 5) until all fuel pins are treated. 


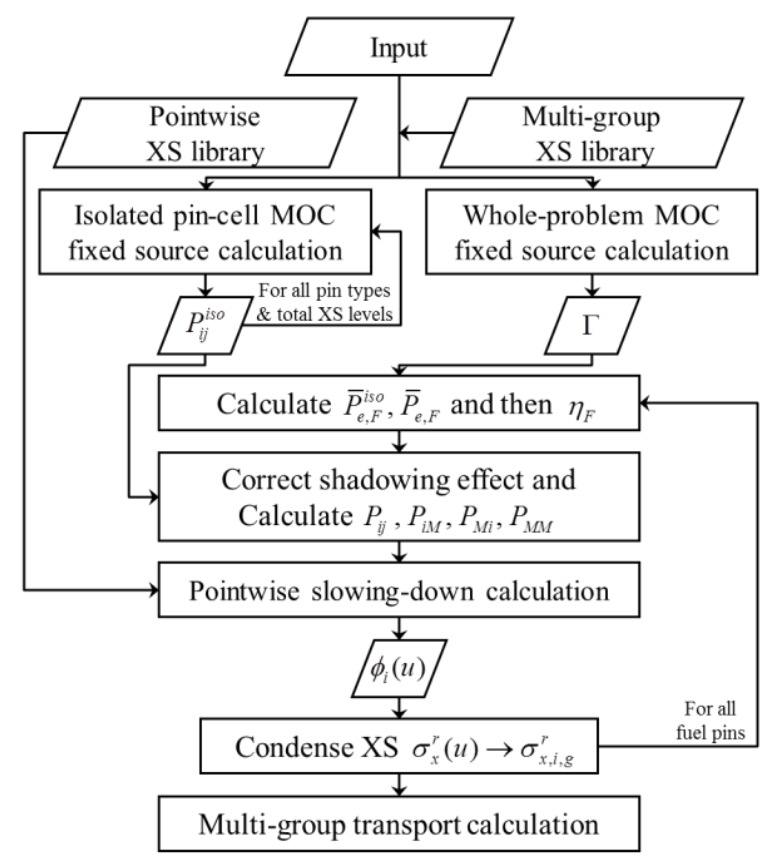

Fig. 10. Flowchart of the pin-based pointwise energy slowing-down solution method.

\section{Verification}

PSM is verified by solving various LWR problems. In Section 4.1, PSM and the contemporary resonance self-shielding methods are compared to a pin-cell problem, and the optimum calculation option of PSM is decided. In Section 4.2, more realistic LWR problems are analyzed with various resonance treatment methods. In Section 4.3, the computation efficiency of PSM is analyzed and compared to the equivalence theory. In all comparisons, reference results are generated by MCNP6, and the STREAM code is used to generate data with various resonance treatment methods. The XS libraries are MCNP6 and STREAM are based on ENDF/B-VII.0 nuclear data. STREAM uses pointwise energy and multi-group XS and RI libraries. Both libraries are generated by using the NJOY code [20]. STREAM uses the 72 energy group structure. For the fast (i.e., $24.78 \mathrm{keV} \sim 20 \mathrm{MeV}$ ) and thermal (i.e., $10^{-5}$ $\mathrm{eV} \sim 0.625 \mathrm{eV}$ ) energy groups, a typical pointwise energy spectrum is used as a weighting spectrum. The microscopic XSs in the fast and thermal energy range depend on a temperature only. The resonance self-shielding calculation is performed to generate a problem-dependent multi-group XS for the resonance energy (i.e., $0.625 \mathrm{eV} \sim 24.78 \mathrm{keV}$ ) range. PSM uses the pointwise energy library to generate the effective XS of fuel, while other methods (i.e., EQ, SDDM, DRI) use the pre-generated RI table to calculate the effective XS. 


\subsection{Base Pin-cell Problem}

In this section, PSM is tested using the base pin-cell problem modeled in Section 2.2. The base pin-cell problem described in Section 2.2 has only one resonant nuclide ${ }^{238} \mathrm{U}$. In the comparison, only absorption XSs of ${ }^{238} \mathrm{U}$ are compared. The problem is solved with various submesh options with PSM. The number of submeshes of the fuel rod varies from 1 to 21 . Its sensitivity result is shown in Fig. 11. The ${ }^{238} \mathrm{U}$ absorption XS is averaged with multi-group flux result as follows:

$$
\bar{\sigma}_{x, g}^{r}=\frac{\sum_{i \in F} \sigma_{x, i, g}^{r} \phi_{i, g} V_{i}}{\sum_{i \in F} \phi_{i, g} V_{i}} .
$$

In Fig. 11, 1-mesh overestimates the ${ }^{238} \mathrm{U}$ absorption XS, especially in groups 10 to 13. The bias in the absorption XS is reduced as the number of meshes is increased. There is no noticeable differences among absorption XSs from 12-mesh to 21-mesh. Based on the result it is concluded that the distribution of the resonance scattering source in the fuel region is very important and thus at least 12 radial submeshes are required to accurately model the effect of resonance scattering. This study specifies that the radial sub-divisions and relevant resonance treatment are required not only to treat the rim effect [1] but also to model the resonance scattering source distribution.

The radial submeshes are usually determined to have the same volume (or $\Delta r^{2}$; where $r$ is radius) for all submeshes. The use of more than 10 radial submeshes is quite expensive in terms of memory and computation time. Therefore, the mesh division is optimized to have the same $\Delta r^{8}$ for all submeshes. This way allows outer rings to have more fine meshes. In Fig. 11, there is no noticeable difference between an optimized mesh (Opt-5-mesh) and 15mesh. The optimized mesh division is used in Section 4.2. 


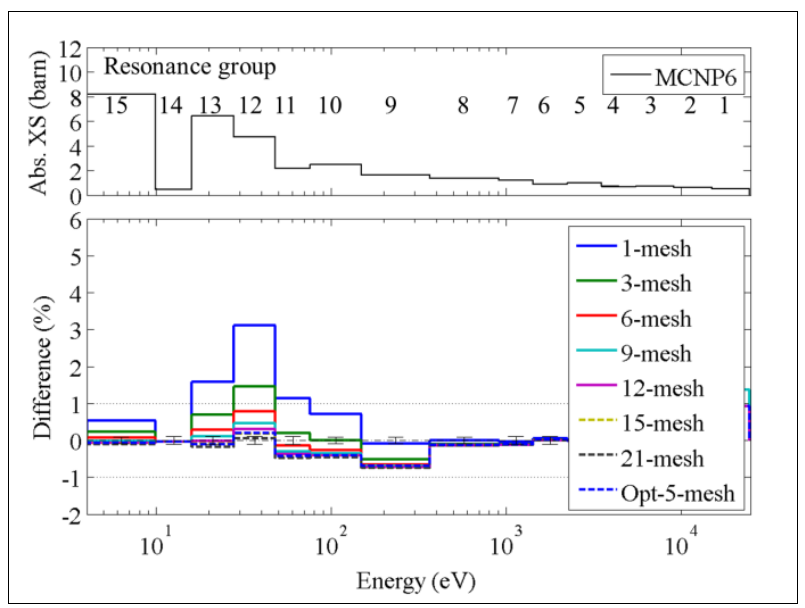

Fig. 11. Comparison of fuel region averaged ${ }^{238} \mathrm{U}$ absorption XS for PSM submesh sensitivity test (base pin-cell problem).

PSM is compared to contemporary resonance self-shielding methods. Following methods are implemented in the STREAM code, as compared in Fig. 12.

1) EQ: Conventional equivalence theory without sub-division in the fuel rod (Section 2.1).

2) DRI: Distributed resonance integral method used in CASMO-5 [10] with 15 submeshes in the fuel.

3) SDDM: Spatially dependent Dancoff method used in PARAGON [11] with 15 submeshes.

4) PSM: Pin-based pointwise slowing-down solution method developed in this paper with 15 submeshes (Sections 3.2 and 3.3)

As discussed in Section 2.2, EQ has a significant bias in ${ }^{238} \mathrm{U}$ absorption XS of groups 9 to 13. Both DRI and SDDM have similar tendencies each other. DRI and SDDM reduce the overestimated absorption XS in groups 9 to 15. However, there are still significant differences, which are less than 4.5\%. DRI and SDDM tend to underestimate the absorption $\mathrm{XS}$ of groups 1 to 8 where the equivalence theory does not have bias. On the other hand, PSM shows a good agreement with MCNP6 solutions. The difference is less than $0.5 \%$, except for the resonance group 1 where unresolved resonances exist. Currently PSM does not 
consider the self-shielding effect on the unresolved resonance and therefore about $1 \%$ difference is observed in the resonance energy group 1. Further study is required to treat the unresolved resonance range. On the other hand, EQ, DRI and SDDM use an RI look-up table generated by the UNRESR module of NJOY. Since, however, the resonance treatment of unresolved resonance range is not important for the LWR application in terms of reactivity and power distribution, PSM without unresolved resonance self-shielding can still provide accurate solutions. The comparison result of reactivity and power distributions is shown in the following sections.

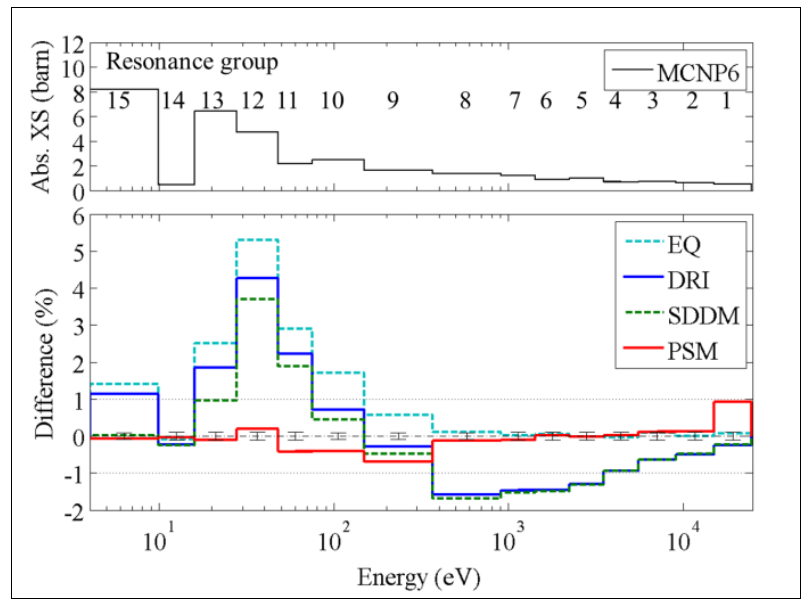

Fig. 12. Comparison of fuel region averaged ${ }^{238} \mathrm{U}$ absorption XS with spatially dependent resonance self-shielding method (base pin-cell problem).

Detailed region-wise absorption XSs of DRI, SDDM and PSM are compared in Fig. 13. The comparison results are shown for energy groups 7, 11 and 12, which show the error behaviors representatively for narrow (Groups $1 \sim 8$ ) and wide (Groups $9 \sim 15$ ) resonances. A relative radius is defined as a ratio of outer radius of annular submeshes to that of a fuel pellet. EQ is not indicated in Fig. 13 because EQ computes the fuel rod averaged absorption XS, not the spatially dependent XS. Although DRI and SDDM show similar averaged absorption XSs to each other in Fig. 12, there are significant differences in the region-wise XSs. DRI has significant difference in the region-wise absorption XS up to $80 \%$, compared to that of MCNP6. DRI underestimates the absorption XS of the inner region of the fuel in the resonance energy group 7. However, the tendency is opposite in groups 11 and 12. It should 
be noted that the result of CASMO-5 would be different to that from the STREAM code because the DRI model was made through an empirical process [10] be suitable for CASMO5. SDDM still has a significant difference in the region-wise absorption XSs. SDDM also underestimates the absorption XS of the inner region of the fuel of group 7. The maximum difference is about $7 \%$. The underestimated absorption XS of inner region of fuel leads to the $\sim 1.5 \%$ difference in region averaged XSs for group 7 in Fig. 12. In the comparison to average absorption XSs, SDDM tends to overestimate XSs in groups 11 and 12. The tendency is also detected in the region-wise XS comparison in Fig. 13. The group 11 and 12 absorption XSs are overestimated in most submeshes by SDDM. As discussed in Section 3.1, SDDM is limited in modeling a spatially distributed resonance XS because the distribution of the resonance scattering source is not properly considered during the resonance treatment. On the other hand, there is no noticeable difference in all results of PSM. The resonance scattering source distribution in submeshes is accurately modeled by PSM based on the collision probability and pointwise energy source calculation.

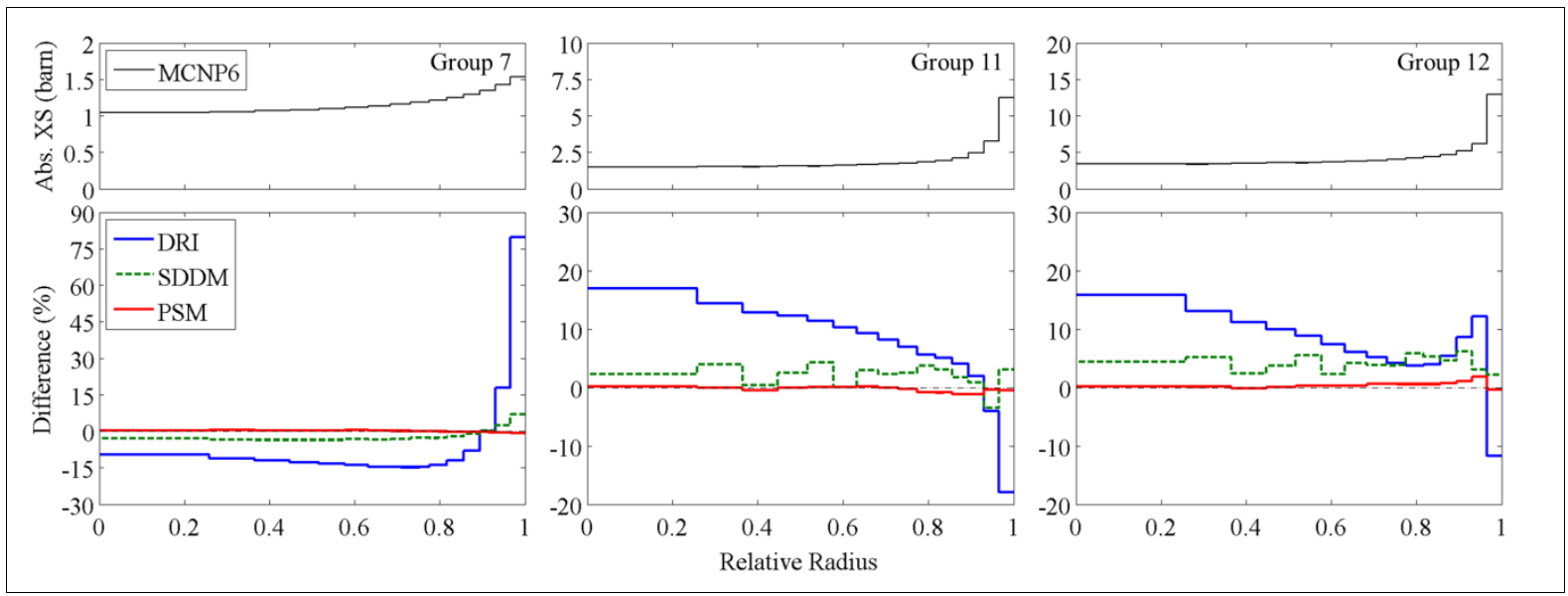

Fig. 13. Comparison of region-wise ${ }^{238} \mathrm{U}$ absorption $\mathrm{XS}$ of resonance groups 7,11 and 12 (base pin-cell problem; relative radius=outer radius of submesh / radius of fuel pellet).

\subsection{Additional Verification Problem}

The base pin-cell problem as described in both Sections 2 and 4.1 is simplified to ignore the errors caused by the resonance interference effect, structure material, or resonance 
upscattering effect. Therefore, in order to solve realistic problems, the methods have to be verified. First, the resonance treatment methods are tested against 62 infinite pin-cell problems as shown in Fig. 14. The cases 1 to 32 are problems from the well-known Mosteller benchmark. Detailed specifications are well described in the reference [21]. The cases 33 to 47 are a burned $\mathrm{UO}_{2}$ fuel problem, and the cases 48 to 62 are burned MOX fuel problems. 5 wt.\% enriched $\mathrm{UO}_{2}$ fuel at hot full power (Case 14) and 8 wt.\% Pu content reactor-recycle MOX fuel at hot full power (Case 24) are depleted up to $60 \mathrm{MWd} / \mathrm{kgU}$ burnup. The depletion calculations are performed with MCNP6, and the material compositions of each burnup step are extracted in order to make STREAM code inputs. More than 120 burnup steps are used in the depletion calculation. However, the 15 steps are selected in order to reduce the number of test models.

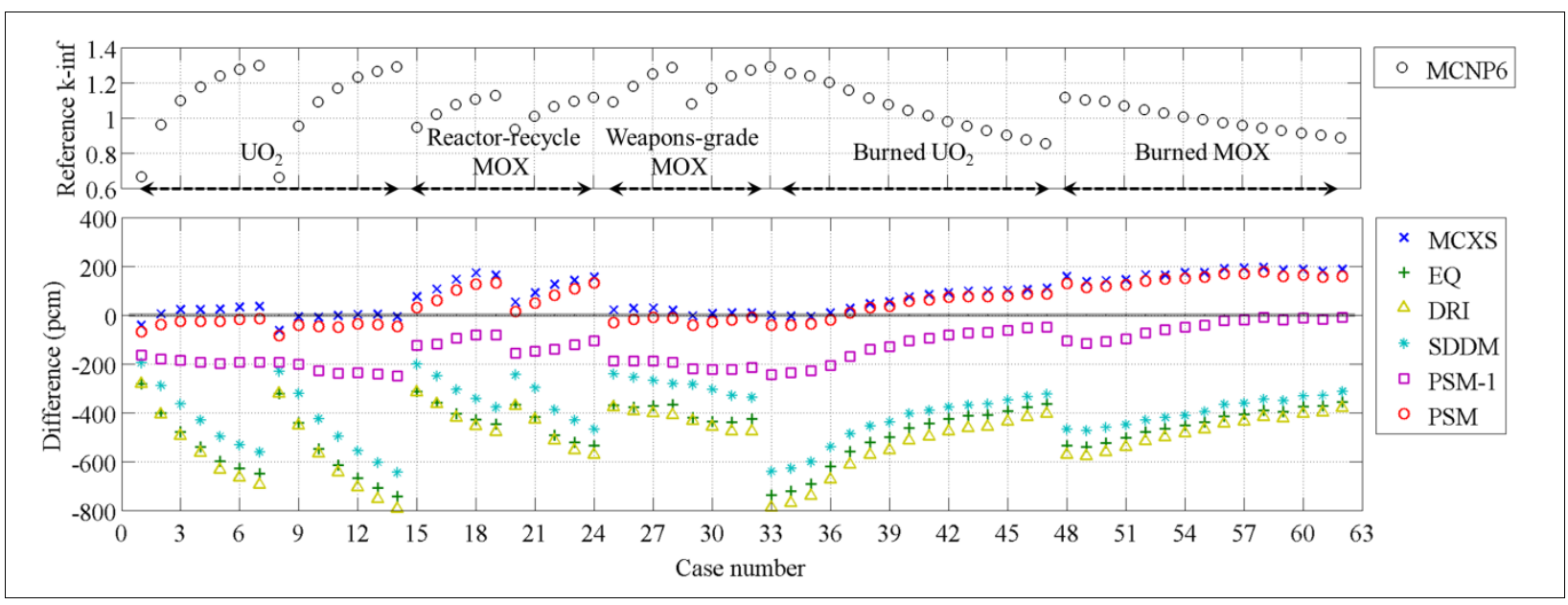

Fig. 14. Pin-cell comparison results.

In Fig. 14, MCXS is the result with the multi-group XSs of resonance energy range (0.3 $\mathrm{eV}$ to $24 \mathrm{keV}$ ) from MCNP6. The multi-group XSs for capture, scattering and fission reactions were tallied by using MCNP6 and then the STREAM code used the XS data for the simulations. Therefore, it can be said that MCXS has no difference in the multi-group XS, compared to MCNP6 results. PSM-1 is PSM without subdivision in fuel pellet regions. The equivalence theory based methods (EQ, DRI and SDDM) show similar results each other. The eigenvalues from the three methods are underestimated by about $200 \sim 800 \mathrm{pcm}$. The first reason for the underestimation is the resonance scattering XS of ${ }^{238} \mathrm{U}$. The second reason 
is the resonance interference effect. The Bondarenko iteration method [2] is used in order to treat the resonance interference effect in the three equivalence-theory methods. It is known that the Bondarenko iteration method causes significant errors in the eigenvalue and resonance XSs [6]. PSM-1 still shows an underestimated eigenvalue by 200 pcm because the resonance scattering source distribution is not properly modeled. PSM shows significant improvement in eigenvalue calculations. PSM has less than $100 \mathrm{pcm}$ differences for the $\mathrm{UO}_{2}$ fuel problems and less than 200 pcm differences for the MOX fuel problems. There is less than $50 \mathrm{pcm}$ difference between PSM and MCXS. This means that the error from the resonance treatment of PSM is less than $50 \mathrm{pcm}$. In other words, the error of less than $50 \mathrm{pcm}$ comes from the difference in the multi-group XS between the PSM and Monte Carlo simulations. The first purpose of the resonance treatment is to calculate an accurate multigroup XS, compared to the continuous energy Monte Carlo result. There are many error sources in multi-group calculations (i.e., discrepancy in the reaction rates from continuous energy calculation and multi-group calculation; errors in thermal and fast energy range). The error from other sources except for the resonance multi-group XS is out of the scope of the resonance treatment.

An additional sensitivity test was performed to examine how the results would change for different ratios of the fuel diameter to pin pitch. A hot zero power pin-cell with $2.4 \mathrm{wt} . \%$ enriched $\mathrm{UO}_{2}$ fuel in the Mosteller benchmark was selected as a base model, and then the fuel diameter was changed from $0.2143 \mathrm{~cm}$ to $0.5143 \mathrm{~cm}$. The corresponding ratio of the fuel diameter to pin pitch varied from 0.3401 to $0.8163 \mathrm{~cm}$. The ratio of the original design was $0.6220 \mathrm{~cm}$. In the variation, the thicknesses of the gap and cladding were fixed to $0.00828 \mathrm{~cm}$ and $0.05746 \mathrm{~cm}$, respectively. The sensitivity test result is in Fig. 15. The differences in the multiplication factor increase as the ratio increases. As the ratio of the fuel diameter to pin pitch increases, the energy and spatial self-shielding effects become larger. The problem with the large ratio is that it also has a significant interference effect. Therefore, a careful resonance treatment should be performed. EQ, DRI and SDDM have significant bias as a function of the diameter to pitch ratio because of the above reasons. The differences of k-inf are $100 \sim 200$ pcms for the small ratio, but they become larger: up to about $600 \mathrm{pcm}$. On the other hand, PSM gives quite accurate and consistent results. PSM has differences in the order of $100 \mathrm{pcm}$ in k-inf. By comparing PSM and PSM-1, it is noted that the effect of the resonance scattering source distribution also becomes larger as the ratio increases because the 
contribution of resonance scattering source increases as the moderator volume decreases. The inaccurate modelling of the resonance scattering source distribution can lead to a difference of up to $200 \mathrm{pcm}$.

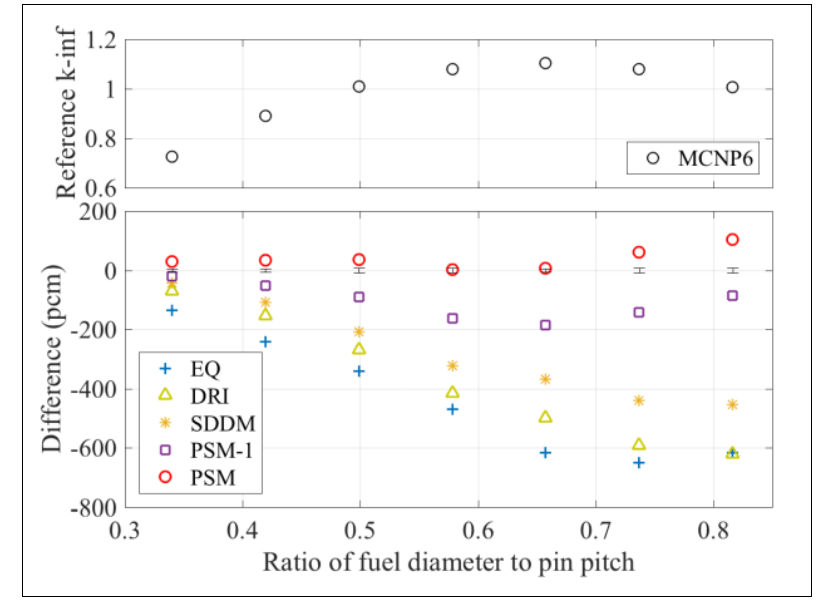

Fig. 15. Sensitivity test result for ratio of fuel diameter to pin pitch.

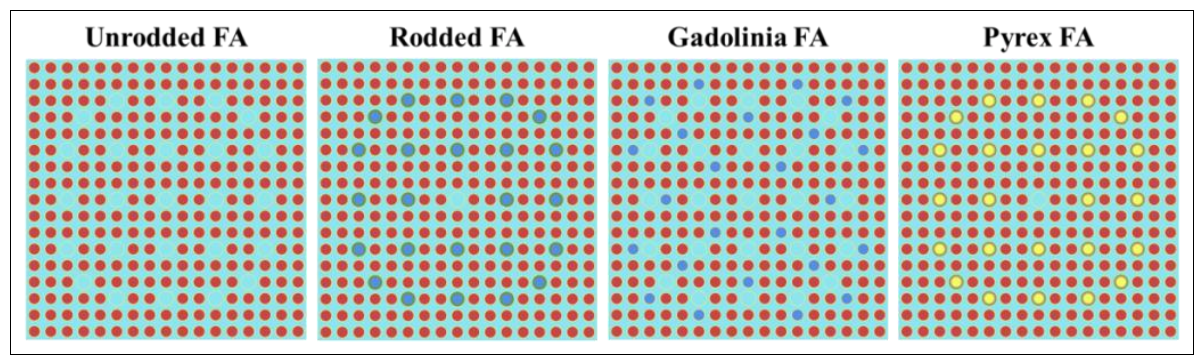

Fig. 16. Configurations of $17 \mathrm{x} 17$ fuel assembly problems

In addition to the infinite pin-cell problems, the resonance treatment methods are known to solve four kinds of $17 \times 17$ fuel assemblies (FA) such as unrodded FA, rodded FA, Gadolinia fuel FA and FA with Pyrex rods as shown in Fig. 16. The four models are same as VERA benchmark problems 2B, 2H, 2P, and 2F, respectively [22]. Detail specifications are described in the VERA benchmark. Similarly to the infinite pin-cell problems, the three equivalence theory methods (EQ, DRI and SDDM) show $200 \sim 600 \mathrm{pcm}$ underestimation in eigenvalue as shown in Table 3 and Table 4. PSM-1 shows $100 \sim 300$ pcm higher eigenvalues than the equivalence theory methods. PSM shows differences in the order of $100 \mathrm{pcm}$ in the 
eigenvalues, compared to MCNP6 results. No noticeable differences in the pin power distributions were observed among the resonance treatment methods.

\section{Table 3}

Unrodded and rodded FA analyses results.

\begin{tabular}{|c|c|c|c|c|c|c|}
\hline \multirow[t]{3}{*}{ Method } & \multicolumn{3}{|c|}{$\begin{array}{l}\text { Unrodded FA } \\
3.1 \text { wt. } \% \mathrm{UO}_{2} \text { fuel }\end{array}$} & \multicolumn{3}{|c|}{$\begin{array}{l}\text { Rodded FA } \\
3.1 \text { wt. } \% \mathrm{UO}_{2} \text { fuel }\end{array}$} \\
\hline & \multirow{2}{*}{$\begin{array}{l}\text { k-inf } \\
\text { difference } \\
(\mathrm{pcm})\end{array}$} & \multicolumn{2}{|c|}{$\begin{array}{l}\text { Pin power } \\
\text { difference }(\%)\end{array}$} & \multirow{2}{*}{$\begin{array}{l}\text { k-inf } \\
\text { difference } \\
(\mathrm{pcm})\end{array}$} & \multicolumn{2}{|c|}{$\begin{array}{l}\text { Pin power } \\
\text { difference }(\%)\end{array}$} \\
\hline & & RMS & Max & & RMS & Max \\
\hline MCNP6 & $\begin{array}{l}1.18309 \\
\pm 4\end{array}$ & \pm 0.07 & \pm 0.09 & $\begin{array}{l}0.78728 \\
\pm 4\end{array}$ & \pm 0.09 & \pm 0.09 \\
\hline EQ & -630 & 0.11 & 0.30 & -264 & 0.16 & 0.40 \\
\hline DRI & -634 & 0.11 & 0.30 & -269 & 0.16 & 0.40 \\
\hline SDDM & -492 & 0.11 & 0.30 & -188 & 0.16 & 0.40 \\
\hline PSM-1 & -158 & 0.16 & 0.50 & 57 & 0.19 & 0.50 \\
\hline PSM & 10 & 0.11 & 0.30 & 158 & 0.17 & 0.50 \\
\hline
\end{tabular}

Table 4

Gadolinia FA and Pyrex FA analyses results.

\begin{tabular}{|c|c|c|c|c|c|c|}
\hline \multirow[t]{3}{*}{ Method } & \multicolumn{3}{|c|}{$\begin{array}{l}\text { Gadolinia FA } \\
3.1 \text { wt. } \% \mathrm{UO}_{2} \text { fuel }\end{array}$} & \multicolumn{3}{|c|}{$\begin{array}{l}\text { Pyrex FA } \\
3.1 \text { wt. } \% \mathrm{UO}_{2} \text { fuel }\end{array}$} \\
\hline & \multirow{2}{*}{$\begin{array}{l}\text { k-inf } \\
\text { difference } \\
(\mathrm{pcm}) \\
\end{array}$} & \multicolumn{2}{|c|}{$\begin{array}{l}\text { Pin power } \\
\text { difference }(\%)\end{array}$} & \multirow{2}{*}{$\begin{array}{l}\text { k-inf } \\
\text { difference } \\
(\mathrm{pcm})\end{array}$} & \multicolumn{2}{|c|}{$\begin{array}{l}\text { Pin power } \\
\text { difference }(\%)\end{array}$} \\
\hline & & RMS & Max & & RMS & Max \\
\hline MCNP6 & $\begin{array}{l}0.92690 \\
\pm 5\end{array}$ & \pm 0.08 & \pm 0.09 & $\begin{array}{l}0.97530 \\
\pm 4\end{array}$ & \pm 0.08 & \pm 0.09 \\
\hline EQ & -583 & 0.11 & 0.30 & -499 & 0.12 & 0.40 \\
\hline DRI & -582 & 0.11 & 0.30 & -505 & 0.12 & 0.40 \\
\hline SDDM & -484 & 0.12 & 0.30 & -395 & 0.12 & 0.40 \\
\hline PSM-1 & -174 & 0.12 & 0.40 & -97 & 0.13 & 0.40 \\
\hline PSM & 8 & 0.12 & 0.40 & 34 & 0.13 & 0.40 \\
\hline
\end{tabular}

\subsection{Comparison of Computing Time}

PSM shows a good agreement in the reactor parameter for the various verification problems. However, it should be confirmed that PSM calculates the multi-group effective XS within a reasonable computation time for the practical use. The unrodded 17x17 FA problem 
is selected for the computation time comparison. Since DRI and SDDM require a very small additional computation cost compared to EQ, only EQ is compared to PSM. The time comparison results are shown in Table 5. In the calculation, any symmetric conditions are not used. Instead, a full geometry is analyzed as is. The results are generated in an OSX system with $3.1 \mathrm{GHz}$ Intel Core i7.

For the computation time comparison, two options are used as follow: 1) Rigorous MOC condition: $0.01 \mathrm{~cm}$ ray distance, 128 azimuthal angles, and T-Y optimized 3 polar angles [23]; 2) Coarse MOC condition: $0.05 \mathrm{~cm}$ ray distance, 48 azimuthal angles, and 3 polar angles. In the test, each pin-cell has 8 azimuthal sectors, 3 radial subregions in the coolant, and 5 radial subregions in the fuel pellet. The inflow transport corrected $\mathrm{P}_{0}\left(\mathrm{TCP}_{0}\right)$ model is used for both of the two options. Generally, the coarse MOC condition and the $\mathrm{TCP}_{0}$ model are used for the practical calculation. It should be noted that all of calculation results in the previous sections were calculated with the rigorous MOC calculation option and $\mathrm{P}_{2}$ high order scattering model to reduce errors from MOC and anisotropic source.

As discussed in Section 3.2, PSM performs energy independent MOC fixed source calculations to consider the shadowing effect. On the other hand, EQ requires 15 MOC fixed source solutions for the fuel treatment. The STREAM code uses 15 resonance energy groups, and each resonance group has its own IR parameter and thus energy group dependent MOC fixed source solutions are required. Actually, the STREAM code has 24 extended resonance energy groups below $4 \mathrm{eV}$ [5]. However, the IR parameter of the resonance group 1 is used for the extended resonance group. Therefore, no additional fixed source problem is solved. For the unrodded 17x17 FA problem, the Dancoff factors are calculated for fuel and clad. PSM is not applied to the resonance treatment for cladding. Both PSM and EQ use an identical resonance treatment method [5] for cladding so that both methods perform energy group dependent MOC fixed source calculations. Therefore, PSM requires 16 MOC fixed source solutions ( 1 for fuel; 15 for clad) while EQ requires 30 fixed source solutions (15 for fuel; 15 for clad). That is why PSM needs about half the computation time in the fixed source calculation (MOC FSP in Table 5) compared to EQ. The time for the MOC FSP depends on the MOC ray conditions.

PSM performs pointwise slowing-down calculations for each individual fuel pin in a given problem. In case of the unrodded 17x17 FA problem, the pointwise flux is calculated 
for the 264 fuel pins. As shown in Table 5, about 4.9 seconds were spent for the pointwise flux calculation of all fuel pins in the problem. PSM also spent less time in using MG XS and RI libraries because PSM did not use RI look-up table to calculate effective XSs of fuel materials.

As a conclusion, the computation time does not matter in PSM. PSM has the advantage of a reduced number of MOC fixed source calculations. When the rigorous MOC ray conditions are used, PSM gives the solution within a shorter time than EQ. Since the elapsed time in the PW energy calculation is less than the elapsed time to get 14 MOC fixed source problem solutions, PSM is more efficient than EQ.

\section{Table 5}

Comparison of computing time in 17x17 FA analysis between EQ and PSM.

\begin{tabular}{|c|c|c|c|c|c|c|c|}
\hline \multirow{2}{*}{$\begin{array}{l}\text { MOC ray } \\
\text { Condition }^{\mathrm{a}}\end{array}$} & \multirow[t]{2}{*}{ Method } & \multicolumn{6}{|c|}{ Elapsed time (sec) } \\
\hline & & $\begin{array}{l}\text { MOC FSP } \\
\text { for fuel }^{b}\end{array}$ & $\begin{array}{l}\text { MOC FSP } \\
\text { for clad }^{c}\end{array}$ & $\begin{array}{l}\text { Using MG } \\
\text { library }\end{array}$ & $\begin{array}{l}\text { PW energy } \\
\text { calculation }\end{array}$ & $\begin{array}{l}\mathrm{XS} \\
\text { total }^{\mathrm{f}}\end{array}$ & Total $^{\mathrm{g}}$ \\
\hline Coarse & EQ & 2.3 & 2.2 & 1.4 & - & 8.1 & 50.9 \\
\hline$(0.05 / 48 / 3)$ & PSM & 0.2 & 2.2 & 0.4 & 4.9 & 8.9 & 51.9 \\
\hline Fine & EQ & 31.5 & 29.8 & 1.4 & - & 64.8 & 627.4 \\
\hline$(0.01 / 128 / 3)$ & PSM & 2.2 & 29.8 & 0.3 & 5.0 & 38.7 & 580.9 \\
\hline \multicolumn{8}{|c|}{ 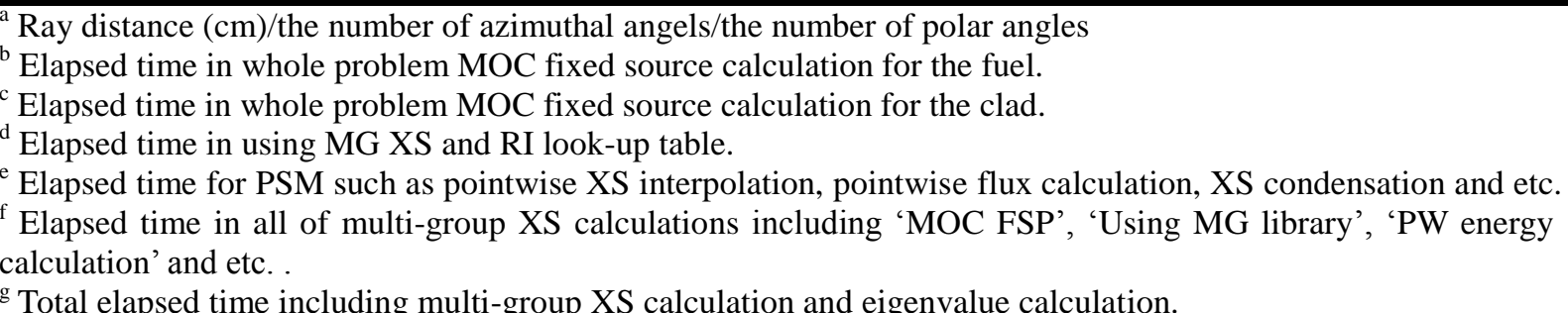 } \\
\hline
\end{tabular}

\section{Discussion}

In Section 4, PSM is tested with various LWR problems, showing high accuracies in the eigenvalue and resonance XSs. However, it should be noted that PSM uses the following assumptions. First, PSM assumes constant material composition and temperature inside the fuel pellet. This is because $P_{i j}^{i s o}\left(\Sigma_{t, i}^{*}\right)$ is generated as a function of the total XS of the fuel lump to avoid energy dependent collision probability calculations. Traditionally, the $\mathrm{UO}_{2}$ fuel is treated as a single region, and the assumption gives reasonable results in terms of reactivity. 
However, the recent trend of the reactor physics requires more detailed information such as the radial distributions of burnup, power, material composition and temperature. PSM can calculate the multi-group effective XS with radially distributed information by using Eqs. (61) and (62) explicitly. However, the underlying assumption can cause an error in the collision probability. Second, when the shadowing effect is considered with the MOC fixed source calculation, it is assumed that all fuel pins in a given problem are identical. This comes from the black pin assumption in the Dancoff factor calculation. It should be noted that the treatment on the shadowing effect with the Dancoff factor is only valid under the black pin condition which is used as one of constraints to compute the rational approximation of the fuel collision probability for a wide range of total XSs (or optical length). The Carlvik's two-term approximation with the Dancoff factor gives a good agreement in the collision probability [5]. However, the Carlvik's two-term equation with the Dancoff factor cannot consider different material compositions between fuel pins in terms of the shadowing effect. The black pin assumption can cause an error when the fuel pins have significantly different fuel materials to each other, such as fuels used in the fast reactor. In this case, the shadowing effect should be calculated for nuclide-by-nuclide [25]. It means the MOC fixed source calculations are required for individual nuclides, which will increse the calculation time. In LWR analyses, however, the black pin assumption still gives an accurate result because similar fuels are used in the reactor core. Many lattice physics codes based on the equivalence theory uses this assumption [1].

PSM has the following advantages compared to the contemporary resonance treatment methods. First, PSM eliminates errors from the resonance scattering source which cannot be treated in the existing equivalence theories. As discussed in Section 2, the resonance scattering causes significant errors in the effective XS. The derivation of the effective XS with the multi-term rational equation and scattering source distribution inside the fuel pellet causes an error when the fuel has a significant resonance scattering XS. Second, the resonance interference effect is considered spontaneously because PSM solves pointwise energy slowing-down equation with the mixed fuel material. Third, PSM can reduce the number of whole-problem MOC fixed source calculations, as described in Section 4.3. This advantage makes it possible for PSM to generate the effective XS faster than the equivalence theory when the MOC fixed source calculation is expensive (i.e., rigorous MOC ray conditions). Fourth, PSM does not need the pre-generated resonance integral library. The 
lattice physics codes based on equivalence theory use the multi-group RI library generated in a homogeneous system using the NJOY code. The codes using subgroup methods or ESSM require a more complicated RI library (or subgroup parameter) which is generated in the heterogeneous system. The library should be generated as a function of a background XS, temperature, and a fuel to moderator volume ratio. On the other hand, PSM uses the multigroup XS library and the pointwise energy XS library. The data of fast and thermal multigroup XSs, scattering matrix, high order scattering XSs, potential scattering XSs, and mass comes from the multi-group XS library. Although the STREAM code with PSM uses the equivalence theory to calculate the effective XS of cladding, PSM can be easily extended to treat the cladding resonance by assuming that fuel does not have resonance. This assumption works well because the cladding does not have a significant resonance self-shielding effect. The RI table of ${ }^{238} \mathrm{U}$ is used to consider the resonance upscattering effect but this is not the main focus of PSM.

\section{Conclusions}

A new resonance self-shielding method, PSM, has been developed in order to eliminate the limitations of the conventional equivalence theory based methods. There are two limitations related to the assumptions in the resonance scattering source. First, the IR approximation in the equivalence theory used for the derivation of the multi-group effective $\mathrm{XS}$, makes it possible to express the flux energy distribution in a heterogeneous system as a combination of those of multiple homogeneous systems. However, if exact resonance scattering sources are used explicitly in the derivation, the heterogeneous system cannot be written as a combination of homogeneous systems. Second, the spatial scattering source distribution inside the fuel pellet is ignored in the equivalence theory, and instead a constant scattering source is assumed in the IR approximation. The above two limitations can cause non-negligible errors in the multi-group XSs around the large scattering resonances.

In this paper, PSM has been newly derived to solve a spatially dependent pointwise energy slowing-down equation for the problem of sub-divided fuel rod and fictitious moderator material. In this method, the collision probabilities within an isolated fuel pellet need to be calculated as a function of total XSs. The shadowing effect correction factor is defined with a two-term rational equation and the Dancoff factor in order to capture the 
spatial self-shielding effect from neighboring fuel rods and structure material. The collision probabilities of the sub-divided fuel rods and moderator can be calculated with the shadowing effect correction factor and the collision probability versus the total XS table of an isolated fuel pin. The pointwise energy slowing-down equation with the calculated collision probabilities is solved for each individual fuel rod in a given problem, and then multi-group XSs are calculated using the pointwise flux solutions and cross sections. The verification calculations with various LWR problems show good agreements between STREAM (with PSM) and MCNP6 in eigenvalue within $100 \mathrm{pcm}$ and in the absorption XSs within $1 \%$. It is also demonstrated that the computation times using PSM are comparable to those with the conventional equivalence theory methods due to the reduction of the total required number of fixed source MOC calculations in the resonance treatment. In conclusion, PSM successfully overcomes the two main limitations in the conventional equivalence theory methods in terms of the resonance scattering source approximation, maintaining similar computation time.

\section{Acknowledgements}

This was also supported by Korea Energy Technology Evaluation and Planning (KETEP), which is funded by the Korean government Ministry of Trade, Industry and Energy (No. 20131610101850).

\section{References}

1. D. Knott, A. Yamamoto, Lattice Physics Computations, in: D.G. Cacuci (Ed.), Handbook of Nuclear Engineering, Springer US, Boston, MA, 2010, pp. 913-1239. DOI:10.1007/978-0-387-98149-9_9.

2. R.J.J. Stamm'ler, M.J. Abbate, Methods of Steady-State Reactor Physics in Nuclear Design, Academic Press, London, 1983.

3. J. Rhodes, K. Smith, D. Lee, CASMO-5 development and applications, in: Proc. ANS Topical Meeting on Reactor Physics (PHYSOR-2006), Vancouver, British Columbia, Canada, Sep. 10-14, 2006.

4. D.J. Powney, T.D. Newton, Overview of the WIMS 9 resonance treatment, SERCO, ANSWERS/WIMS/TR. 26 Issue 1, 2004. 
5. S. Choi, H. Lee, S.G. Hong, D. Lee, Resonance self-shielding methodology of new neutron transport code STREAM, J. Nucl. Sci. Technol. 52 (2015) 1133-1150. DOI:10.1080/00223131.2014.993738.

6. S. Choi, A. Khassenov, D. Lee, Resonance self-shielding method using resonance interference factor library for practical lattice physics computations of LWRs, J. Nucl. Sci. Technol. (2015) 1-13. DOI:10.1080/00223131.2015.1095686.

7. L. Cao, Q. Zhang, H. Wu, Y. Zheng, An Improved Resonance Self-Shielding Calculation Method Based on Equivalence Theory, Nucl. Sci. Eng. 179 (2015) 233252. DOI:10.13182/NSE13-108.

8. A. Yamamoto, T. Endo, H. Koike, Improved Derivation of Multigroup Effective Cross Section for Heterogeneous System by Equivalence Theory, Nucl. Sci. Eng. 168 (2011) 75-92. DOI:10.13182/NSE10-50.

9. C.C. Stoker, Z.J. Weiss, Spatially dependent resonance cross sections in a fuel rod, Ann. Nucl. Energy. 23 (1996) 765-778. DOI:10.1016/0306-4549(95)00074-7.

10. Z. Xu, J. Rhodes, K. Smith, CASMO-5 versus MCNP-5 Benchmark of Radial Power Profile in a Fuel Pin, in: International Conference on Mathematics, Computational Methods \& Reactor Physics (M\&C 2009), New York, May 3-7 2009.

11. H. Matsumoto, M. Ouisloumen, T. Takeda, Development of Spatially Dependent Resonance Shielding Method, J. Nucl. Sci. Technol. 42 (2012) 688-694. DOI:10.1080/18811248.2004.9726438.

12. A. Yamamoto, Evaluation of Background Cross Section for Heterogeneous and Complicated Geometry by the Enhanced Neutron Current Method, J. Nucl. Sci. Technol. 45 (2012) 1287-1292. DOI:10.1080/18811248.2008.9711916.

13. T. Goorley, M. James, T. Booth, F. Brown, J. Bull, L.J. Cox, J. Durkee, J. Elson, M. Fensin, R.A. Forster, J. Hendricks, H.G. Hughes, R. Johns, B. Kiedrowski, R. Martz, S. Mashnik, G. McKinney, D. Pelowitz, R. Prael, J. Sweezy, L. Waters, T. Wilcox, T. Zukaitis, Initial MCNP6 Release Overview, Nucl. Technol. 180 (2012) 298-315. DOI:10.13182/NT11-135.

14. Y. Liu, W. Martin, M. Williams, K.-S. Kim, A Full-Core Resonance Self-Shielding 45 / 47 
Method Using a Continuous-Energy Quasi-One-Dimensional Slowing-Down Solution that Accounts for Temperature-Dependent Fuel Subregions and Resonance Interference, Nucl. Sci. Eng. 180 (2015) 247-272. DOI:10.13182/NSE14-65.

15. K.S. Kim, M.L. Williams, D. Wiarda, T. Andrew, Development of a New 47-Group Library for the CASL Neutronics Simulator, in: ANS MC2015 - Joint International Conference on Mathematics and Computation (M\&C), Supercomputing in Nuclear Applications (SNA) and the Monte Carlo (MC) Method, Nashville, TN, April 19-23, 2015.

16. I. Carlvik, A method for calculating collision probabilities in general cylindrical geometry and applications to flux distributions and Dancoff factors, in: Third United Nations International Conference on the Peaceful Uses of Atomic Energy, Geneva, Switzerland, August 31-September 9, 1964.

17. P.H. Kier, A.A. Robba, RABBLE: A Program for Computation of Resonance Absorption in Multiregion Reactor Cells., Argonne National Laboratory, ANL-7326, 1967. DOI:10.2172/4245065

18. D. Lee, K. Smith, J. Rhodes, The impact of ${ }^{238} \mathrm{U}$ resonance elastic scattering approximations on thermal reactor Doppler reactivity, Annals of Nuclear Energy. 36 (2009) 274-280. DOI:10.1016/j.anucene.2008.11.026.

19. Y. Li, Q. He, L. Cao, H. Wu, T. Zu, Resonance Elastic Scattering and Interference Effects Treatments in Subgroup Method, Nucl. Eng. Technol. 48(2) (2016) 339-350. http://dx.doi.org/10.1016/j.net.2015.12.015

20. A.C. Kahler, R.E. MacFarlane, D.W. Muir, R.M. Boicourt, The NJOY Nuclear Data Processing System, Version 2012, Los Alamos National Laboratory, LA-UR-1227079, 2012.

21. R.D. Mosteller, The Doppler-defect benchmark: overview and summary of results, in: Joint International Topical Meeting on Mathematics \& Computation and Supercomputing in Nuclear Applications (M\&C + SNA 2007), Monterey, California, April 15-19, 2007.

22. A. T. Godfrey, VERA Core physics benchmark progression problem specifications. 
Rev. 3, Oak Ridge National Laboratory, CASL-U-2012-0131-003, 2014.

23. A. Yamamoto, M. Tabuchi, N. Sugimura, T. Ushio, M. Mori, Derivation of Optimum Polar Angle Quadrature Set for the Method of Characteristics Based on Approximation Error for the Bickley Function, J. Nucl. Sci. Technol. 44 (2012) 129136. DOI:10.1080/18811248.2007.9711266.

24. S. Choi, K. Smith, H.C. Lee, D. Lee, Impact of inflow transport approximation on light water reactor analysis, J. Comput. Phys. 299 (2015) 352-373. DOI:10.1016/j.jcp.2015.07.005.

25. C.H. Lee, W.S. Yang, An improved resonance self-shielding method for heterogeneous fast reactor assembly and core calculations, in: International Conference on Mathematics and Computational Methods Applied to Nuclear Science and Engineering (M\&C 2013), Sun Valley, Idaho, May 5-9, 2013 